\title{
Railway wheel profile fine-tuning system for profile recommendation
}

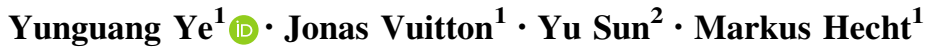

Received: 8 December 2020/Revised: 28 January 2021 / Accepted: 29 January 2021/Published online: 7 March 2021

(C) The Author(s) 2021

\begin{abstract}
This paper develops a wheel profile fine-tuning system (WPFTS) that comprehensively considers the influence of wheel profile on wheel damage, vehicle stability, vehicle safety, and passenger comfort. WPFTS can recommend one or more optimized wheel profiles according to train operators' needs, e.g., reducing wheel wear, mitigating the development of wheel out-of-roundness (OOR), improving the shape stability of the wheel profile. Specifically, WPFTS includes four modules: (I) a wheel profile generation module based on the rotary-scaling finetuning (RSFT) method; (II) a multi-objective generation module consisting of a rigid multi-body dynamics simulation (MBS) model, an analytical model, and a rigidflexible MBS model, for generating 11 objectives related to wheel damage, vehicle stability, vehicle safety, and passenger comfort; (III) a weight assignment module consisting of an adaptive weight assignment strategy and a manual weight assignment strategy; and (IV) an optimization module based on radial basis function (RBF) and particle swarm optimization (PSO). Finally, three cases are introduced to show how WPTFS recommends a wheel profile according to train operators' needs. Among them, a wheel profile with high shape stability, a wheel profile for mitigating the development of wheel OOR, and a wheel profile considering hunting stability and derailment safety are developed, respectively.
\end{abstract}

Yunguang Ye

yunguang.ye@campus.tu-berlin.de

1 Institute of Land and Sea Transport Systems, Technical University of Berlin, 10587 Berlin, Germany

2 College of Transportation Science and Engineering, Nanjing Tech University, Nanjing 210009, China
Keywords Wheel profile fine-tuning system · Optimization · Recommendation - Wear · Contact concentration index $\cdot$ Multi-body dynamics simulation (MBS) · Railway wheel

\section{Introduction}

A reasonable railway wheel profile contributes to improving vehicle-track dynamic performance, prolonging the service life of wheelsets, or reducing maintenance costs. Optimizing the wheel profile, therefore, has been a topic of concern to train operators and interested scholars since the dawn of railway vehicles [1]. The wheel profile optimization methods proposed in the past two decades have been briefly reviewed in Ref. [1]. In summary, these methods with different strategies fall into two categories: (I) singleobjective optimization methods and (II) multi-objective optimization methods.

Classical single-objective optimization methods include target contact angle method [2], target rolling radius difference (RRD) method [3, 4], target conicity method [5], target normal gap method [6], etc. These methods can maximize or minimize a single specific objective (e.g., wear), but cannot comprehensively consider the influence of wheel profile on different dynamic parameters that affect running stability, curve-negotiation performance, and maintenance-related costs, and whose relationships may be competing. For instance, the running stability is usually contradictory to the curve-negotiation performance [5]. Multi-objective optimization methods based on multi-body dynamics simulation (MBS), therefore, have attracted more 
attention in recent years due to their ability to balance different dynamic performances where competing relationships exist. In addition to a rigid MBS model, the multi-objective optimization methods mainly consist of two submodules: (1) a wheel profile generation method for producing candidate profiles and (2) an optimization algorithm for automatically finding an optimal profile.

Regarding the wheel profile generation method, the most commonly used is the method based on fitting discrete points as presented in Ref. [7]. In this method, the wheel profile is considered to be composed of an optimization region and a non-optimization region. The points in the optimization region are vertically movable, while the points in the non-optimization region are fixed, i.e., corresponding to the default profile. Another classical method is the element combination method [8]. In this method, the wheel profile is divided into several elements (e.g., straights, arcs, and splines), and one or more elements act as the optimization region, where the geometric parameters (e.g., length and radius) of the active element are changeable. These two methods can generate a large number of candidate profiles but, meanwhile, faces two challenges: (a) some primary constraints are required to initially ensure the monotonicity and concavity and convexity of the profile curve. After fitting by methods such as NURBS (nonuniform rational B-spline) [10], secondary constraints are further required to ensure the rationality of the final generated profile. However, even under the control of these strict primary and secondary constraints, it is still difficult to ensure that all generated profiles are reasonable, which brings problems for automatic optimization, and an additional manual evaluation is often necessary. (b) They are computationally expensive due to two issues: One is that a large number of discrete points (design variables) means a large number of iterative calculations and MBS runs, and the other is that the strict constraints mentioned in (a) would cause a long convergence time for wheel profile generation [6]. Therefore, in this work, we introduce the RSFT method proposed in Ref. [1] to fine-tune the baseline wheel profile. Based on the baseline profile, the RSFT method can efficiently and quickly produce a large number of candidate profiles with different equivalent conicities and different flange thicknesses by introducing two design variables.

Optimization algorithms used in wheel profile optimization mainly consist of bio-inspired optimization algorithm (e.g., genetic algorithm (GA) [9], particle swarm optimization (PSO) [10], and covariance matrix adaptation evolution strategy (CMA-ES) [11]) and response surface technique (e.g., Kriging surrogate model (KSM) [12]). Among them, the bio-inspired algorithms require a large number of iterative calculations, and the response surface technique has regression capability. How to combine the two methods to quickly and reliably complete the task of optimizing wheel profiles remains to be explored.

The contribution of this paper mainly includes:

- A wheel profile fine-tuning system (WPFTS) is developed, which consists of four modules: (I) a wheel profile generation module, (II) a multi-objective generation module, (III) a weight assignment module, and (IV) an optimization module. This system comprehensively considers the influence of the wheel profile on wheel damage, vehicle stability, vehicle safety, and passenger comfort, and can recommend one or more optimized wheel profiles according to train operators' needs.

- Based on the Manchester Benchmark passenger vehicle, three cases are introduced to demonstrate how WPTFS recommends a wheel profile. Among them, a wheel profile with high shape stability, a wheel profile for mitigating the development of wheel OOR, and a wheel profile considering hunting stability and derailment safety are developed, respectively.

The remaining of this paper is structured as follows. Section 2 briefly introduces the architecture of WPFTS. Section 3 introduces more especially the applied methodology for each module included in WPFTS. Three cases introduced in Sect. 4 show how WPFTS recommends a wheel profile according to train operators' needs. This paper ends with conclusions and discussion.

\section{Architecture of WPFTS}

The developed WPFTS (see Fig. 1) consists of four modules:

I. Wheel profile generation module This module is used for producing candidate profiles for optimization purposes, where the RSFT method proposed in Ref. [1] is used.

II. Multi-objective generation module This module consists of three submodules that run in parallel:

a. An MBS model for generating tread wear index $w_{\mathrm{t}}$, flange wear index $w_{\mathrm{f}}$, wheel surface fatigue $f_{\text {su }}$, wheel-rail vertical force $Q$, sum of wheel-rail lateral forces $H$, derailment coefficient $f_{\text {de }}$, overturning coefficient $\eta$, mean comfort index $N_{\mathrm{mv}}$, and train critical speed $v_{\mathrm{cr}}$.

b. A geometrical analytical model for producing contact concentration index $(\mathrm{CCI})$ proposed by Polach [13].

c. A rigid-flexible coupled MBS model for calculating wheel radial wear deviation (RD). 


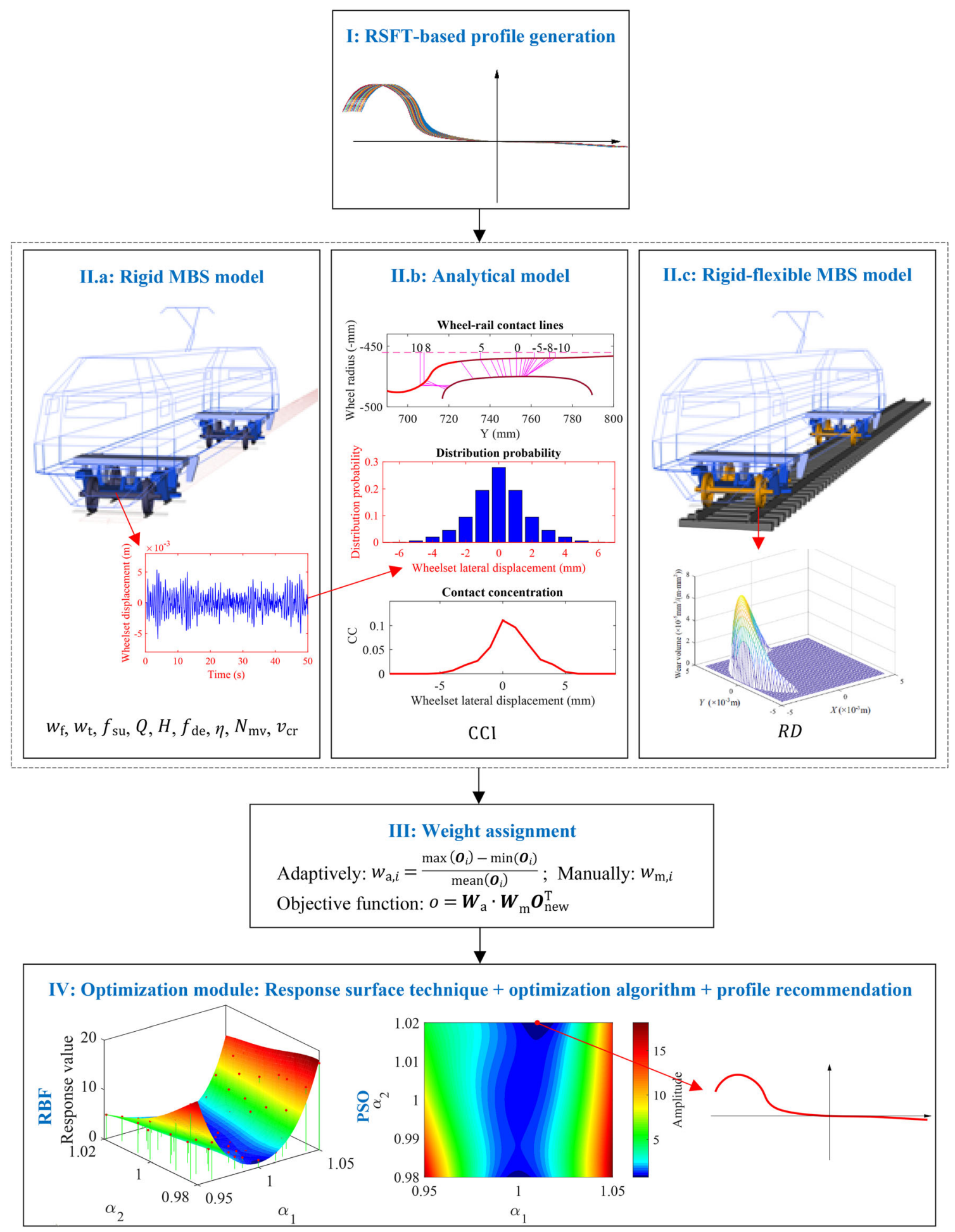

Fig. 1 Architecture of the proposed WPFTS

III. Weight assignment module In this module, an adaptive weight assignment strategy taking into account the sensitivity of an optimization objective against changes of the wheel profile and a manual weight assignment strategy based on the train operators' specific requirements are presented.

IV. Optimization module In this module, a response surface technique (i.e., radial basis function, RBF) and a bio-inspired optimization algorithm (i.e., 
particle swarm optimization, PSO) are combined to quickly and reliably recommend an optimal wheel profile according to train operators' needs.

\section{Processing methodology of WPFTS}

\subsection{Wheel profile generation module}

As mentioned in Sect. 1, the commonly used wheel profile generation methods include the method based on fitting discrete points [7] and the element combination method [8], but they face two challenges: they are computationally expensive, and the generated wheel profiles may be unreasonable. Therefore, in Ref. [1], a comparably conservative RSFT method was proposed, which introduces two design variables $\left(\alpha_{1}\right.$ and $\left.\alpha_{2}\right)$ and an empirical correction formula $(E x)$, to fine-tune the baseline wheel profiles for optimization purposes. In this method, the original profile is defined as a function of the lateral position $y$, i.e., $z(y)$, the baseline profile being specified as points in the $(y, z)$ plane. A transformation matrix $\boldsymbol{T}_{1}$ is introduced to rotate the curve until the vertex $A\left(y_{\theta}, z_{\max }\right)$ of the curve coincides with the $O x$-axis, as shown in Fig. 2. The point of coordinates $(y, z)$ after being rotated is written as $\left(y_{1}, z_{1}\right)$ :

$$
\left[\begin{array}{l}
y_{1} \\
z_{1}
\end{array}\right]=\boldsymbol{T}_{1}\left[\begin{array}{l}
y \\
z
\end{array}\right] \text {, and } \boldsymbol{T}_{1}=\left[\begin{array}{cc}
\cos \theta & \sin \theta \\
-\sin \theta & \cos \theta
\end{array}\right]
$$

where $\theta=\arctan \left(z_{\max } / y_{\theta}\right)$ is the rotation angle, $z_{\max }$ and $y_{\theta}$ are the ordinate and abscissa of point $A$, respectively. The curve $z(y)$ after being rotated is written as $z_{1}(y)$, and a correction coefficient $\alpha_{1}$ is introduced to scale the $z$ coordinate of the points on the curve: $\left[\begin{array}{l}y_{2} \\ z_{2}\end{array}\right]=\left[\begin{array}{c}y_{1} \\ \alpha_{1} z_{1}\end{array}\right]$

A transformation matrix $\boldsymbol{T}_{2}=\boldsymbol{T}_{1}^{\mathrm{T}}$, which is used to rotate the curve $z_{2}(y)$ back, is introduced to generate the curve $z_{3}(y)$, and the generated points $\left(y_{3}, z_{3}\right)$ of $z_{3}(y)$, corresponding to $(y, z)$ of $z(y)$, is expressed as

$\left[\begin{array}{l}y_{3} \\ z_{3}\end{array}\right]=\boldsymbol{T}_{2}\left[\begin{array}{l}y_{2} \\ z_{2}\end{array}\right]$.

An empirical correction function $E x$ is introduced to modify the curve, and the generated curve is written as $z_{4}(y)$. The dotted red line in Fig. 2 shows the obtained empirical correction function $E x$ for $\alpha_{1}=0.95$.

$$
\begin{aligned}
& {\left[\begin{array}{l}
y_{4} \\
z_{4}
\end{array}\right]=\boldsymbol{T}_{2}\left[\begin{array}{c}
y_{2} \\
z_{2}-E x
\end{array}\right],} \\
& E x=\sin \left(\frac{\pi}{2} \frac{y_{3}}{\left|y_{\theta}\right|}\right)\left(z_{3}-z\right) .
\end{aligned}
$$

Then, the other correction factor $\alpha_{2}$ to scale the $y$ coordinates of these points $\left(y_{4}, z_{4}\right)$ on the curve $z_{4}(y)$ is introduced to fine-tune the flange thickness, and the final curve is expressed as

$$
\left[\begin{array}{l}
y_{5} \\
z_{5}
\end{array}\right]=\left[\begin{array}{c}
\alpha_{2} y_{4} \\
z_{4}
\end{array}\right] \text {. }
$$

To ensure that the generated wheel profile satisfies the requirements (e.g., flange thickness, height, flange slope quota, maximum contact angle) specified in standards such as EN 15,313 [14], $\alpha_{1}$ is set between $0.95 \leq \alpha_{1} \leq 1.05$, and $\alpha_{2}$ is set between $0.98 \leq \alpha_{2} \leq 1.08$ for freight vehicles and $0.98 \leq \alpha_{2} \leq 1.02$ for passenger vehicles for the profile S1002. The detailed theory and MATLAB codes of the RSFT method were disclosed in Ref. [1].

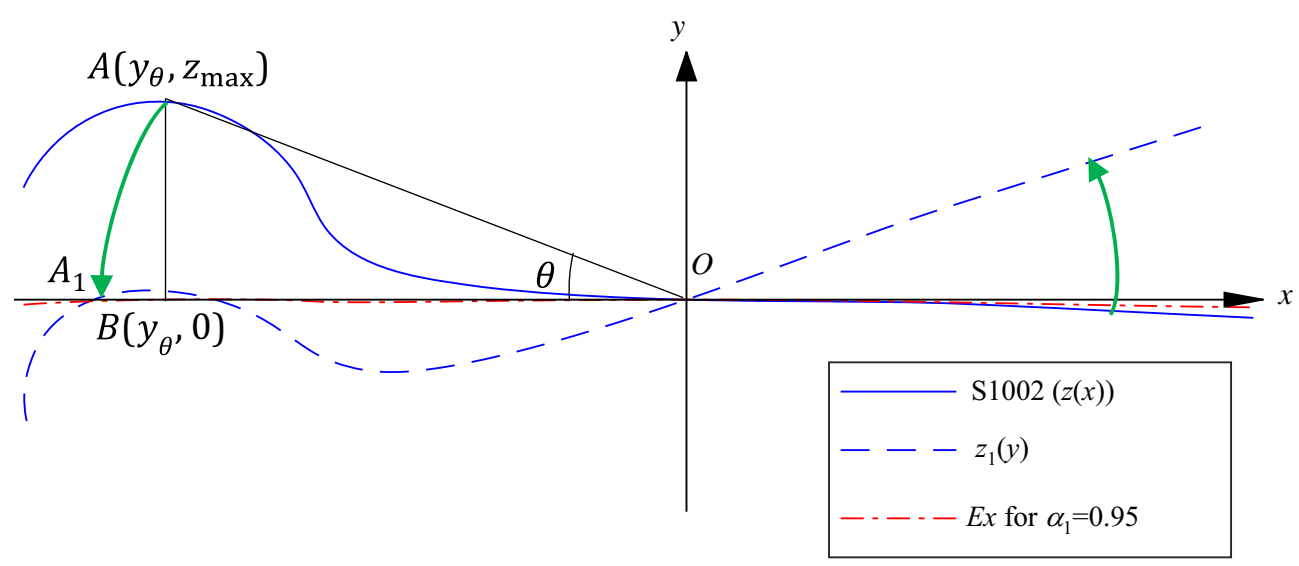

Fig. 2 Rotation step and empirical correction function $E x$ for $\alpha_{1}=0.95$ of the RSFT method 


\subsection{Multi-objective generation module}

\subsubsection{Rigid MBS model}

3.2.1.1 Vehicle running on a virtual track In the rigid MBS model shown in Fig. 1, the Manchester Benchmark passenger vehicle [15] with a constant speed of $200 \mathrm{~km} / \mathrm{h}$ running on a $15-\mathrm{km}$ virtual track with the layout data listed in Table 1 is used to generate eight objectives, including $w_{\mathrm{t}}, w_{\mathrm{f}}, f_{\mathrm{su}}, Q, H, f_{\mathrm{de}}, N_{\mathrm{mv}}$, and $\eta$, where the track irregularities measured from a high-speed railway line are used, as shown in Fig. 3. The rail profile is 60E2 [16], the rail cant is $1 / 40$, the track gauge is $1435 \mathrm{~mm}$, and the gauge measurement position is $14 \mathrm{~mm}$ below the rail crown.

The formula for calculating wear index is

$$
w=\int_{s_{\text {start }}}^{s_{\text {end }}}\left(F_{x} \gamma_{x}+F_{y} \gamma_{y}\right) \mathrm{d} s,
$$

Table 1 The virtual track used in the rigid MBS model

\begin{tabular}{llll}
\hline $\begin{array}{l}\text { Radius } \\
(\mathrm{m})\end{array}$ & $\begin{array}{l}\text { Length of } \\
\text { transition } \\
\text { curve }(\mathrm{m})\end{array}$ & $\begin{array}{l}\text { Length of circular } \\
\text { curved track }(\mathrm{m})\end{array}$ & $\begin{array}{l}\text { Superelevation } \\
(\mathrm{mm})\end{array}$ \\
\hline 5000 & 360 & 600 & 120 \\
5500 & 360 & 1150 & 165 \\
7000 & 360 & 1200 & 145 \\
8000 & 340 & 1350 & 120 \\
9000 & 300 & 1184 & 100 \\
12,000 & 220 & 1093 & 80 \\
$\infty$ & - & 6600 & - \\
\hline
\end{tabular}

where $F_{x}$ and $F_{y}$ are the longitudinal and lateral creep forces, respectively; $\gamma_{x}$ and $\gamma_{y}$ are the longitudinal and lateral creepages, respectively; $s_{\text {start }}$ and $s_{\text {end }}$ are the start distance and end distance, respectively. Freight trains are characterized by heavy axle load, and their routes often contain many small-radius curves. The flange wear is often severe. Therefore, the wear index is divided into tread wear index $w_{\mathrm{t}}$ and flange wear index $w_{\mathrm{f}}$. The definition of the tread region and flange region of the S1002/h28/e32.5/ $6.7 \%$ wheel profile specified in standard EN 13,715 [17] is shown in Fig. 4:

- Flange region, between H2 and D1, the point D1 being defined as the start point of flange on the profile;

- Connection zone, between D1 and $\mathrm{C} 1 *$, the point $\mathrm{C} 1 *$ being defined on the profile at a lateral position of $26 \mathrm{~mm}$;

- Tread region, between $\mathrm{C} 1^{*}$ and $\mathrm{B} 1^{*}$, the point $\mathrm{B} 1^{*}$ being defined as the connection point of the reverse slope with the wheel tread;

- Field zone, between B1* and I.

However, in the present work, the connection zone D1$\mathrm{C} 1 *$ is allocated to the flange region, while the field zone $\mathrm{B} 1 *-\mathrm{I}$ to the tread region. The newly defined flange region and tread region are $\mathrm{H} 2-\mathrm{C} 1 *$ and $\mathrm{C} 1{ }^{*}-\mathrm{I}$, respectively. This is due to the fact that the wear index is used to characterize the wear level, but when the wheel-rail contact occurs in the flange (H2-D1) and connection zone (D1-C1*), the contact patch is much smaller than that in the tread $\left(\mathrm{C} 1 *_{-}\right.$ $\mathrm{B} 1 *)$ and field zone $\left(\mathrm{B} 1{ }^{*}-\mathrm{I}\right)$, resulting in a disproportionate relationship between material loss and wear index and more serious material loss in the flange and connection (a)

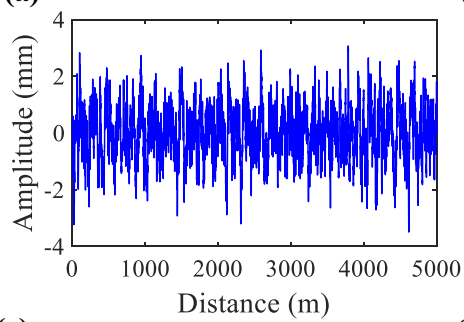

(c)

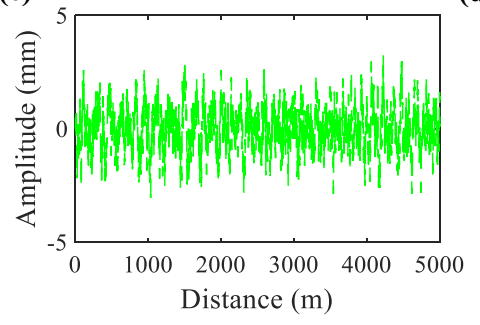

(b)

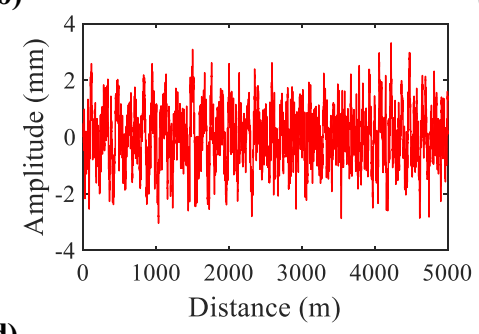

(d)

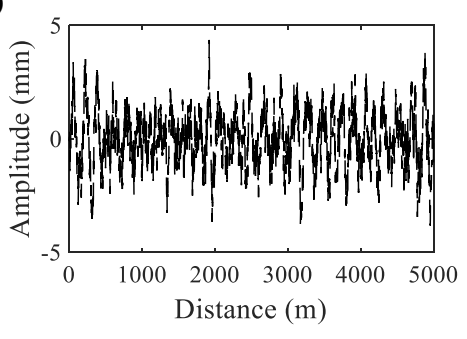

(e)

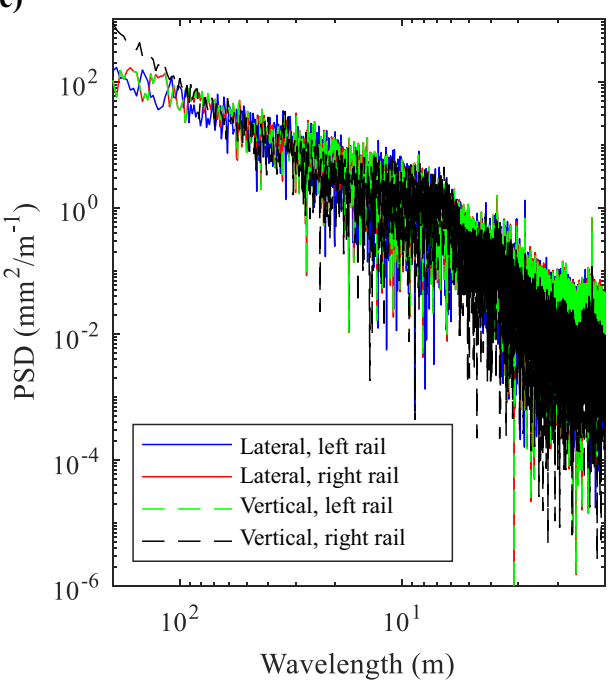

Fig. 3 Measured irregularities from a high-speed railway line: a left rail (lateral), b right rail (lateral), c left rail (vertical), d right rail (vertical), and $\mathbf{e}$ their power spectrum densities 
zone [18]. Considering the sum of the wear index in the regions $\mathrm{H} 2-\mathrm{C} 1 *$ and $\mathrm{C} 1{ }^{*}-\mathrm{I}$ as the optimization objective would underestimate the damage in the region $\mathrm{H} 2-\mathrm{C} 1 *$, this conclusion will be verified in Sect. 4.1.

The formula for calculating the wheel surface fatigue index is as follows [19, 20]:

$f_{\mathrm{su}}=\int_{s_{\mathrm{start}}}^{s_{\mathrm{end}}}\left(\mu-\frac{2 \pi a b k}{3 F_{\mathrm{n}}}>0\right) \mathrm{d} s, \mu=\frac{\sqrt{F_{x}^{2}+F_{y}^{2}}}{F_{\mathrm{n}}}$,

where $\mu$ is the traction coefficient; $a$ and $b$ are the semiaxes of the Hertzian contact, respectively; $F_{x}, F_{y}$, and $F_{\mathrm{n}}$ are the longitudinal, lateral, and normal forces, respectively; $k=300 \mathrm{MPa}$ is the yield limit in cyclic shear.

Wheel-rail vertical force $Q$, sum of wheel-rail lateral forces $H$, derailment coefficient $f_{\text {de }}$, and overturning coefficient $\eta$ with the following formula are used to evaluate the performance of a wheel profile $[20,21]$ :

$$
\left\{\begin{array}{l}
Q_{\max }<90(\mathrm{kN})+Q_{0}(\mathrm{kN}) \\
(H)_{\max }=\left(\sum Y_{2 \mathrm{~m}}\right)_{\max }<10(\mathrm{kN})+\frac{P_{0}}{3}(\mathrm{kN}) \\
\left(f_{\mathrm{de}}\right)_{\max }=\left[\left(\frac{Y}{Q}\right)_{2 \mathrm{~m}}\right]_{\max }<0.8 \\
\eta_{\max }=\left(\frac{\sum_{\text {bogie }} Q_{i, 1}-\sum_{\text {bogie }} Q_{i, \mathrm{r}}}{\sum_{\text {bogie }} Q_{i, 1}+\sum_{\text {bogie }} Q_{i, \mathrm{r}}}\right)_{\max }<1
\end{array},\right.
$$

where $Q_{0}$ is the static wheel load, $(\cdot)_{2 \mathrm{~m}}$ means sliding mean over $2 \mathrm{~m}$ of track, $P_{0}$ is the static axle load, $Y$ is the wheelrail lateral force, and $Q_{i, 1}$ and $Q_{i, \mathrm{r}}$ are the vertical wheelrail force on the left and right side of the $i$ th wheelset, respectively.

Mean comfort index $N_{\mathrm{mv}}$ This parameter is used to evaluate the ride comfort of passenger vehicles. The formula for calculating this parameter specified in EN 12,299 [22] is expressed as

$$
N_{\mathrm{mv}}=6 \times \sqrt{\left(a_{x, \mathrm{p} 95}^{W_{\mathrm{d}}}\right)^{2}+\left(a_{y, \mathrm{p} 95}^{W_{\mathrm{d}}}\right)^{2}+\left(a_{z, \mathrm{p} 95}^{W_{\mathrm{b}}}\right)^{2}}
$$

First, the CCI as proposed by Polach [13] is presented, which is a theoretical, analytical methodology. In the

where $a_{i, \mathrm{p} 95}^{w_{j}}$ represents the 95th percentile of five-second weighted RMS values of the acceleration on the floor of the vehicle calculated over a time period of five minutes; the subscripts $x, y$, and $z$ represent the longitudinal, lateral, and vertical direction, respectively; the superscript $W_{\mathrm{d}}$ and $W_{\mathrm{b}}$ relate to the weighted frequency values [22] in accordance with the weighting curve in the longitudinal and lateral directions, and the vertical direction, respectively.

In this work, the parameters $w_{\mathrm{t}}, w_{\mathrm{f}}, f_{\mathrm{su}}, Q, H$, and $f_{\mathrm{de}}$ are calculated for the first wheelset running on the virtual track, the parameter $\eta$ for the first bogie, and the parameter $N_{\mathrm{mv}}$ for the whole vehicle. The pre-processing method of the data obtained from the simulation is performed according to standards [21, 22].

\subsubsection{Vehicle running on a straight track Critical speed} $v_{\mathrm{cr}}$ is obtained using the method of decreasing velocity [23] with an initial speed of $400 \mathrm{~km} / \mathrm{h}$ and an acceleration of $0.1 \mathrm{~m} / \mathrm{s}^{2}$ [24]. The vehicle runs on a straight track without considering track irregularities, but a lateral excitation with an amplitude of $4 \mathrm{~mm}$ and a length of $30 \mathrm{~m}$ is set on the track at $50 \mathrm{~m}$ away from the starting position to excite lateral instability of the vehicle.

To obtain the critical speed automatically, the Hilbert envelope [25] of the lateral wheelset displacement is first calculated, and then the speed corresponding to the threshold $y_{\mathrm{WS}}=0.5 \mathrm{~mm}$ is regarded as the critical speed. For a systematic study on the deceleration method for calculating the critical speed, see Ref. [24]. Figure 5 shows the critical speed of the Manchester benchmark passenger vehicle with S1002 wheel profiles obtained automatically using the deceleration method and the Hilbert envelope.

\subsubsection{Analytical model: MBS-based contact concentration index}

,

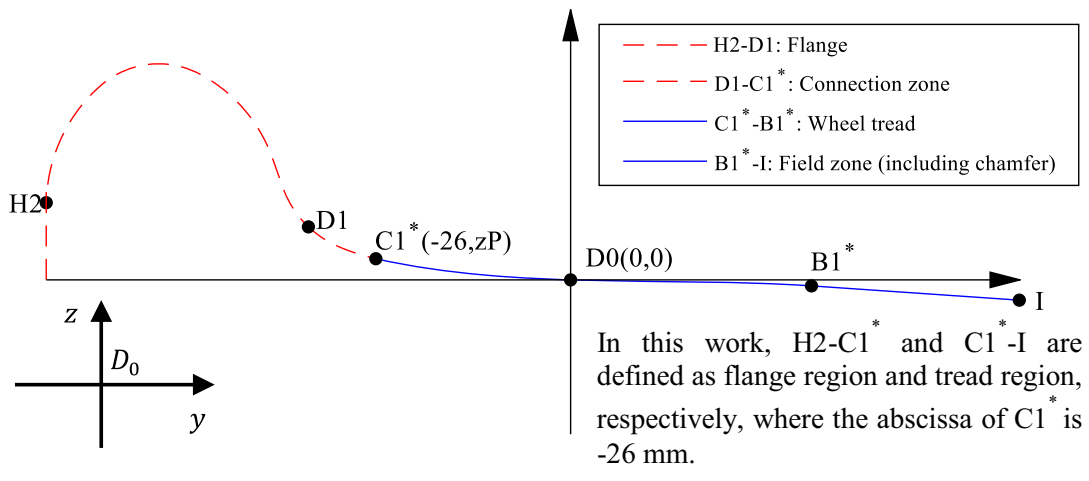

Fig. 4 Definition of the flange region and tread region [17] 
proposed WPFTS, the methodology is enhanced with MBSs to take into account more realistic wheelset lateral displacement.

\subsubsection{Contact concentration index (CCI) High wheel-} rail contact conformity can make the wear distribution of the wheel more uniform, and the profile shape remains more stable, thereby extending the service life of wheelsets. Based on this concept, Polach and Nicklisch [13] proposed the CCI to assess the contact conformity. In Ref. [13], a long-time measurement campaign on German ICE 2 wheel profiles and a comparison between five different new wheel profiles (including two wear-adapted profiles and three non-wear-adapted profiles) have shown the relationship between the CCI and the contact conformity, a small CCI meaning a high contact conformality. Based on Refs. $[5,13,26]$, the calculation steps of CCI, as shown in Fig. 6a, are summarized as follows.

Step 1: Contact point movement $\mathrm{d} y_{\mathrm{C}}\left(y_{\mathrm{WS}}\right)$.

The relationship between the lateral wheelset displacement $y_{\mathrm{WS}}$ and the contact point movement $\mathrm{d} y_{\mathrm{C}}$ is established, where $\mathrm{d} y_{\mathrm{C}}$ represents the derivation of the function of the contact point location on the wheel profile $y_{\mathrm{C}}=f\left(y_{\mathrm{WS}}\right)$, it is calculated as

$$
\mathrm{d} y_{\mathrm{C}}\left(y_{\mathrm{WS}}\right)=\left|\frac{\Delta y_{\mathrm{C}}\left(y_{\mathrm{WS}}\right)}{\Delta y_{\mathrm{WS}}}\right|=\left|\frac{y_{\mathrm{C}}\left(y_{\mathrm{WS}}+\Delta y_{\mathrm{WS}}\right)-y_{\mathrm{C}}\left(y_{\mathrm{WS}}\right)}{\Delta y_{\mathrm{WS}}}\right| \text {. }
$$

The parameters $\Delta y_{\mathrm{WS}}$ and $\Delta y_{\mathrm{C}}\left(y_{\mathrm{WS}}\right)$ are shown in Fig. 6a and represent the change of lateral wheelset displacement and the shift of the contact point position, respectively. In this work, the trace line method proposed by Wang [27] is used to calculate the contact point movement $\mathrm{d} y_{\mathrm{C}}\left(y_{\mathrm{WS}}\right)$, and the wheel-rail contact lines calculated by Wang's method is shown in Fig. $6 \mathrm{~b}$.

Step 2: Normal distribution hypothesis $\varphi_{\sigma}\left(y_{\mathrm{WS}}\right)$.

The following two assumptions are introduced for vehicles running on straight tracks:

- Stochastic track irregularities are distributed.

- The lateral wheelset displacement conforms to Gaussian normal distribution.

Therefore, the occurrence probability of the wheelset displacement is expressed as

$\varphi_{\sigma}\left(y_{\mathrm{WS}}\right)=\frac{1}{\sqrt{2 \pi} \sigma} \exp \left(-\frac{\left(y_{\mathrm{WS}}-\mu\right)^{2}}{2 \sigma^{2}}\right)$,

where $\mu=0$ is the mean of the normal distribution; $\sigma$ is the standard variance, a large value means that the distribution range of the wheelset lateral displacement is wide and vice versa. As in Ref. [13], $\sigma$ is set to 2.5 (the blue line shown in Fig. 6c).

Step 3: Contact concentration $\mathrm{CC}\left(y_{\mathrm{WS}}\right)$.

It is assumed that the local wear of wheels and rails is related to the local frequency of contact point occurrence because wear is higher in the area with a more frequent contact occurrence [13]. Moreover, a wide spread (i.e., low concentration) of the contact points between wheel and rail is usually correlated with conformal contact and thus larger contact patch size, lower contact stress, and consequently lower wear and vice versa, supporting the assumption that wear is proportional to the concentration of the contact

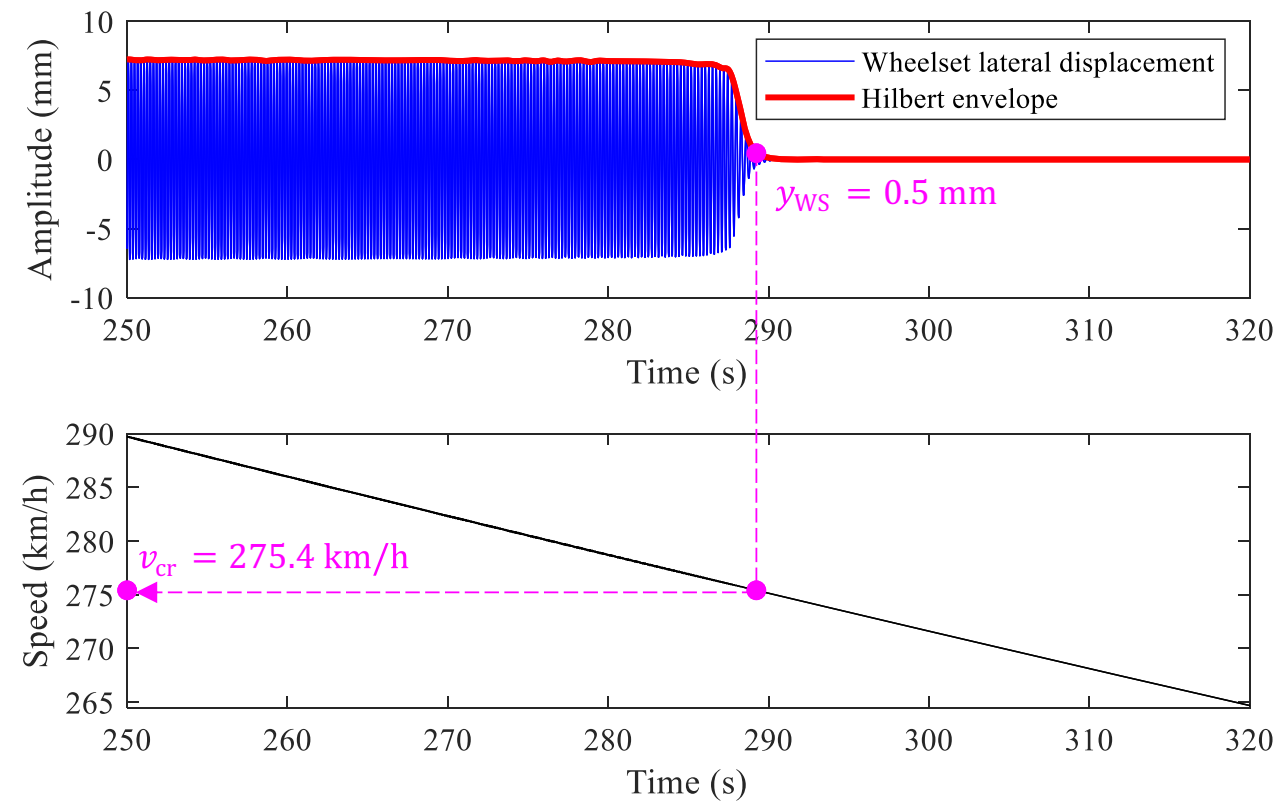

Fig. 5 Automatic acquisition of critical speeds using the Hilbert envelope method 
Step 1:
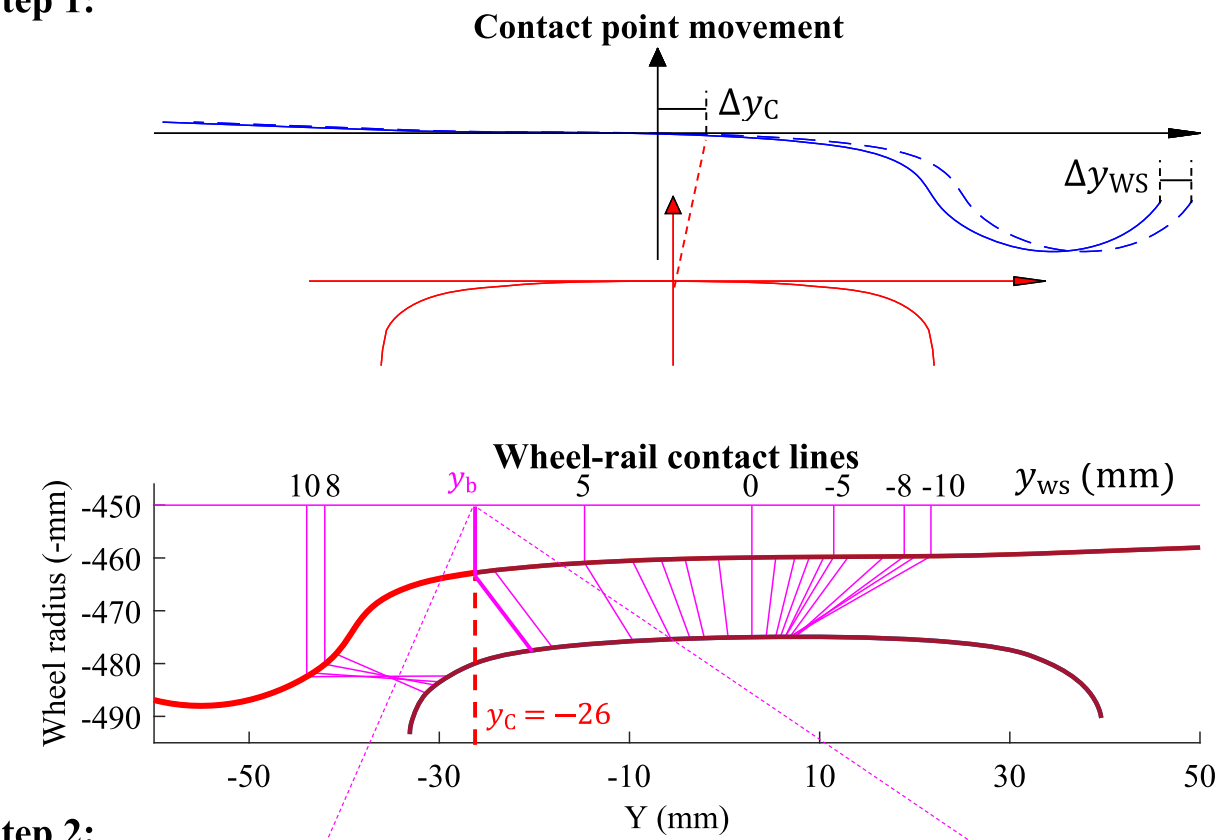

Step 2:

Distribution probability

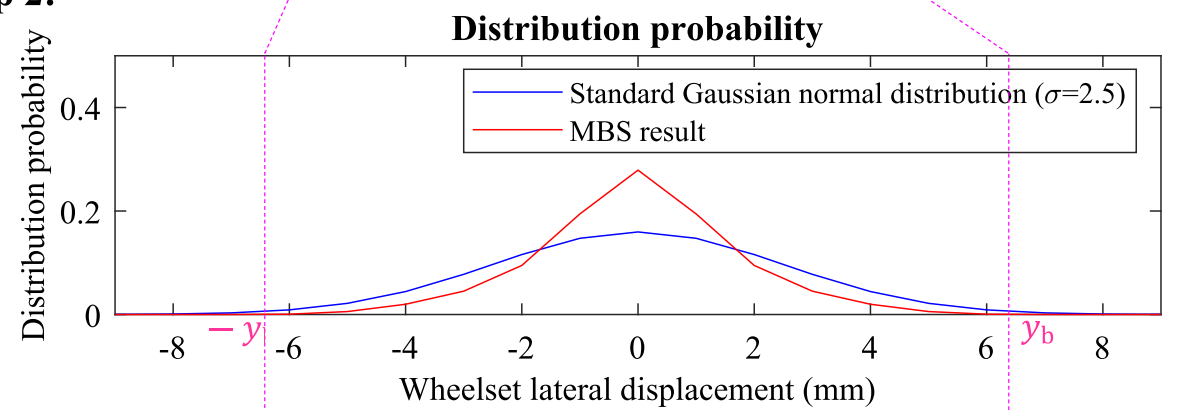

Step 3:

Contact concentration

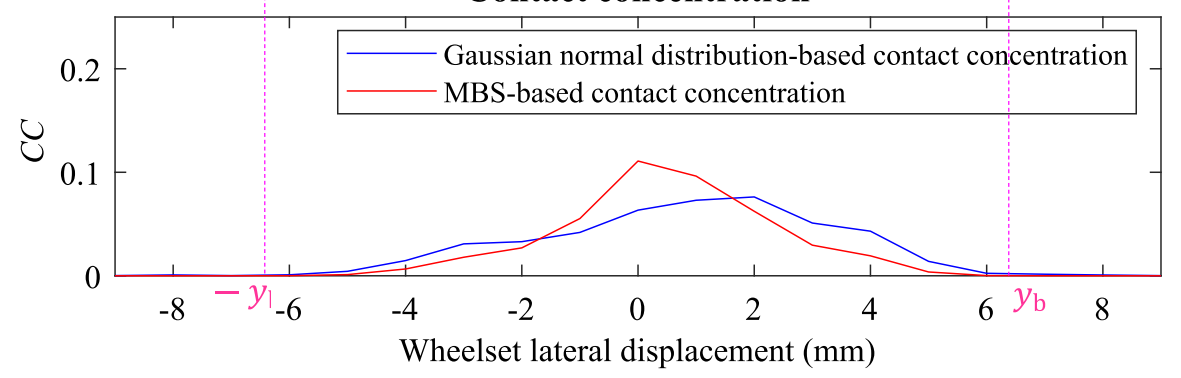

\section{Step 4:}

$$
\text { Polach: } \mathrm{CCI}=\frac{1}{6 \sigma} \int_{-3 \sigma}^{3 \sigma} C C\left(y_{\mathrm{Ws}}\right) \mathrm{d} y_{\mathrm{Ws}} \text {; enhanced: } \mathrm{CCI}=\frac{1}{2 y_{\mathrm{b}}} \int_{-y_{\mathrm{b}}}^{y_{\mathrm{b}}} C C\left(y_{\mathrm{Ws}}\right) \mathrm{d} y_{\mathrm{Ws}}
$$

Fig. 6 Technique diagram of CCI calculation

point occurrence. Based on this concept, the parameter contact concentration $\mathrm{CC}\left(y_{\mathrm{WS}}\right)$ is defined as

$$
\mathrm{CC}\left(y_{\mathrm{Ws}}\right)=\frac{\varphi_{\sigma}\left(y_{\mathrm{WS}}\right)}{\mathrm{d} y_{\mathrm{C}}\left(y_{\mathrm{WS}}\right)} \text {. }
$$

Step 4: Contact concentration index CCI.
To characterize the average contact concentration of different wheel-rail matching forms, CCI is calculated by averaging the contact concentration $\mathrm{CC}\left(y_{\mathrm{WS}}\right)$ over the normal distribution between $-3 \sigma$ and $3 \sigma$ of lateral wheelset displacement [26] as 


$$
\mathrm{CCI}=\frac{1}{6 \sigma} \int_{-3 \sigma}^{3 \sigma} C C\left(y_{\mathrm{Ws}}\right) \mathrm{d} y_{\mathrm{Ws}}
$$

This parameter represents the concentration of contacts and thus the concentration of wear for different wheel-rail matching forms can be evaluated. A large CCI means a poorer contact concentration and vice versa.

\subsubsection{MBS-based CCI In this work, Step 2 of CCI is} enhanced as follows:

- In Refs. [5, 13, 26], it is assumed that the lateral wheelset displacement conforms to Gaussian normal distribution. This approach is efficient, but it may deviate from reality. Therefore, we replace the Gaussian distribution with the distribution obtained by dynamic simulations.

- In the calculation of CCI, the integration interval (lateral wheelset displacement) depends on the predefined standard variance $\sigma$, and a reasonable $\sigma$ depends on the expertise of the researcher. This paper avoids this problem with the help of the MBS result.

Figure $6 \mathrm{c}$ shows the difference between the assumed Gaussian distribution probability $(\sigma=2.5)$ used in Ref. [13] and an MBS-based distribution probability obtained by performing probability statistics on the wheelset lateral displacement simulated over the entire virtual track $(15 \mathrm{~km})$. It can be seen that the Gaussian distribution assumption deviates from the simulated result.

The correctness of CCI also depends on the following assumption in Step 3: It is assumed that the local wear is related to the local frequency of contact point occurrence. The basis of this assumption is that there is a nearly linear relationship between the wheel material loss and the local frequency of contact point occurrence. However, this assumption is usually not reliable when the wheel-rail contact occurs in the flange region, because of the following reasons: (1) the wheel material loss is disproportionate to the contact point occurrence due to large creepage and pressure occurring in the flange region [18]; (2) it may involve multi-point contact that does not follow the contact distribution probability obtained in Step 2. Therefore, as mentioned in Ref. [13], CCI is only applicable to wheels traveling on lines with predominantly straight tracks. Considering the above issues, this paper only calculates the CCI when the wheel-rail contact point locates in the tread region where the lateral position on the wheel profile is greater than $-26 \mathrm{~mm}$ as shown in Fig. 4. Finally, the equation for calculating the MBS-based CCI is rewritten as
$\mathrm{CCI}=\frac{1}{2 y_{\mathrm{b}}} \int_{-y_{\mathrm{b}}}^{y_{\mathrm{b}}} C C\left(y_{\mathrm{WS}}\right) \mathrm{d} y_{\mathrm{WS}}$,

where $y_{\mathrm{b}}$ corresponds to the lateral displacement of the wheelset when the contact happens at a lateral position on the wheel profile of $-26 \mathrm{~mm}$, corresponding to the boundary between the tread and the flange region, as shown in Step 4 of Fig. 6.

\subsubsection{Rigid-flexible MBS model}

This model is mainly used for mitigating the wheel OOR, which is an inevitable problem in the operation of railway vehicles. For a comprehensive literature review concerning OOR of railway wheels, see Refs. [28, 29]. The theoretical basis of this submodule is mainly based on the following findings. Kalousek and Johnson [30] investigated the rail and wheel corrugation problems encountered by the Vancouver mass-transit system. Their findings indicated that a tight conformity of wheel/rail profiles could trigger the growth of corrugations due to the generation of 'roll-slip' oscillation of the wheel-rail system. Morys [31] established an iterative long-term wear model to analyze the enlargement of wheel polygonization phenomena of an ICE1 carriage. Simulation results showed that the bending oscillation of the wheelset axle caused the wheel lateral slip and lateral material loss to exhibit periodic unevenness. Tao et al. [32] pointed out that the lateral slip between the wheels and rails caused by the elastic vibration of the wheelset played a key role in the formation of high-order polygonal wear.

The above findings show that the development of wheel OOR is affected by wheel-rail contact behavior (creepage). On the other hand, an optimized wheel profile can improve contact behavior, which has also been observed in many simulations run for the present study. Based on this consideration, this model is used to mitigate the development of wheel OOR from the perspective of designing the wheel profile. The evaluation index used in this module is the maximum deviation of the wheel's circumferential wear (wheel OOR curve in Fig. 7), i.e., the maximum radial deviation RD.

In the evolution of wheel OOR, the wheelset's elastic deformation plays a key role. In addition, much literature reports that some elements (e.g., sleeper, fastener) in the track system and the flexibility of the rail also have an important influence [33-35]. Therefore, a modal analysis on the wheelset and the track in FEM software has been performed, and the normal modes of the wheelset below $1280 \mathrm{~Hz}$ and the normal modes of the track below $600 \mathrm{~Hz}$ are considered in the model implementation in SIMPACK to establish a rigid-flexible coupling dynamics model (see 

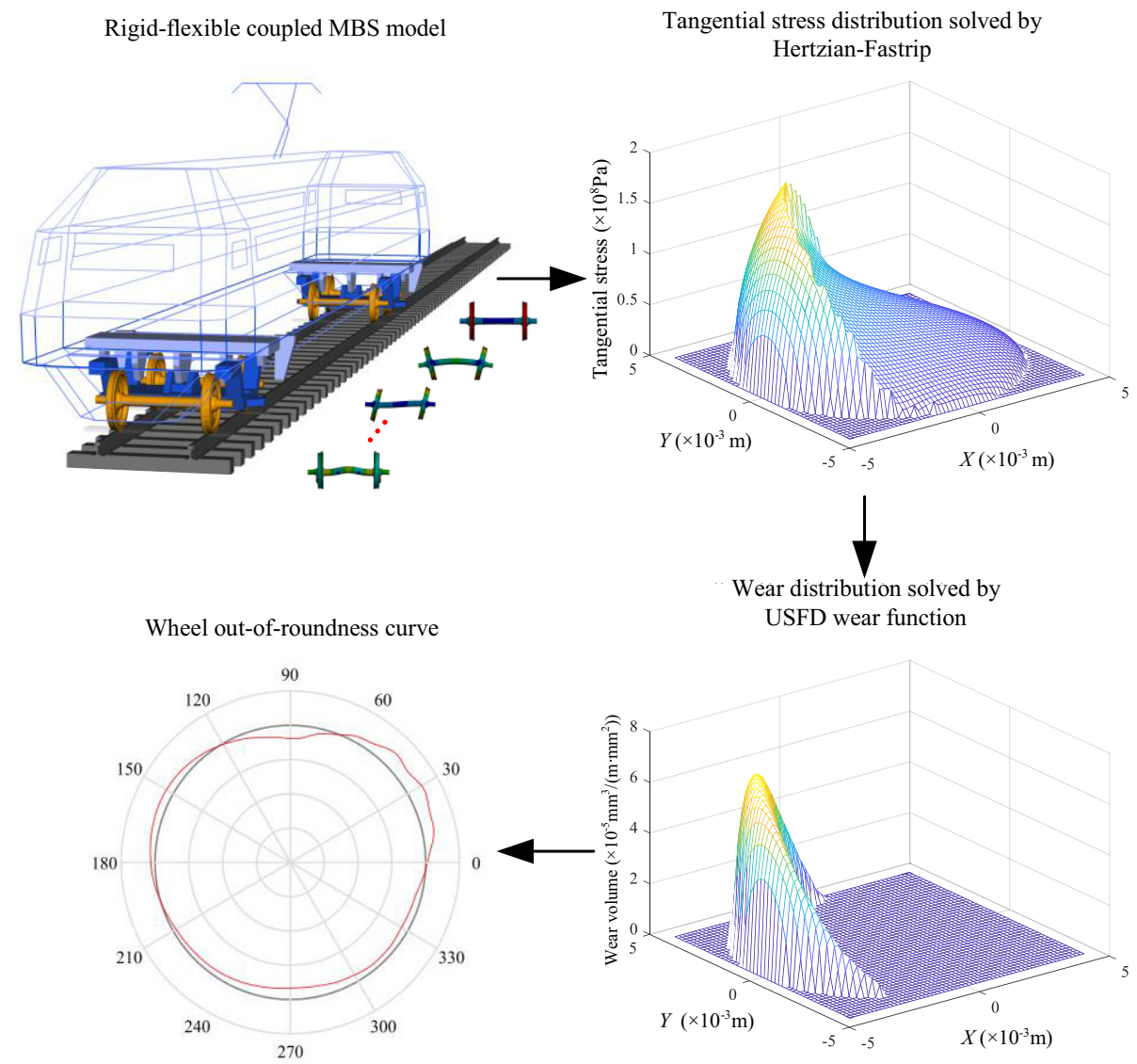

Fig. 7 Technique diagram of wheel OOR calculation. The USFD wear function is the wheel material loss function developed by University of Sheffield (USFD)

Fig. 7), in which four flexible wheelsets are used. Because of the time-intensive simulation process, the vehicle model in this paper only runs on a straight track with a length of 10 wheel revolutions. The randomness of track irregularities in such a short distance is not strong, and it may erroneously cause a specific wavelength of the wheel OOR curve. Therefore, track irregularities are not considered in the rigid-flexible MBS model. The initial wheel radial difference curve was measured from an actual wheel. For the wheel circumferential wear calculation method, as well as more related information including the flexible bodies, see Ref. [36].

\subsection{Weight assignment module}

For a multi-objective optimization problem, it is usually necessary to assign a weight to each objective to transform it into a single-objective optimization problem. Most of the current weight assignment methods are based on expertise and are manually realized. This section introduces an adaptive + manual weight assignment method, in which the adaptive weight assignment strategy takes into account the degree of influence of wheel profile changes on each optimization objective, and the manual weight assignment strategy can establish an objective function according to train operators' needs.

\subsubsection{Adaptive weight assignment strategy}

The change of wheel profile may have a very large influence on some objectives, while the influence on other objectives may not be obvious. For instance, Fig. 8 shows the derailment coefficient and critical speed values corresponding to 79 different wheel profiles; it shows that the change of the wheel profile has a very large influence on the derailment coefficient and the maximum difference can reach $208 \%$, while that for the critical speed is only about $62.33 \%$. To take into account the degree of influence of wheel profile changes on each optimization objective, an adaptive weight adjustment factor $w_{\mathrm{a}}$ is introduced:

$w_{\mathrm{a}, i}=\frac{\max \left(\boldsymbol{O}_{i}\right)-\min \left(\boldsymbol{O}_{i}\right)}{\operatorname{mean}\left(\boldsymbol{O}_{i}\right)}$, 


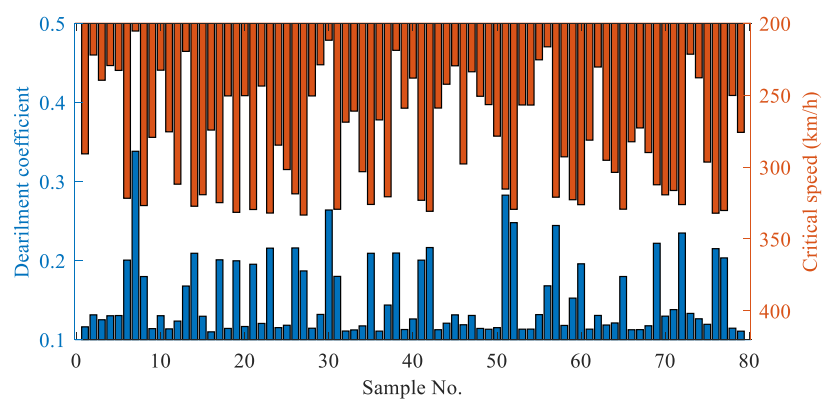

Fig. 8 The simulated derailment coefficient and critical speed

where $\boldsymbol{O}_{i}$ represents the $i$ th objective vector composed of $n=79$ samples. $\max \left(\boldsymbol{O}_{i}\right), \min \left(\boldsymbol{O}_{i}\right)$, and mean $\left(\boldsymbol{O}_{i}\right)$ are the maximum, minimum, and mean values in the vector $\boldsymbol{O}_{i}$, respectively. In other words, the adaptive weight adjustment factor $w_{\mathrm{a}}$ takes into account the sensitivity of an optimization objective against changes of the wheel profile. A larger value means that the objective is more sensitive to changes in wheel profile, and should be considered more in wheel profile optimization.

Then, the min-max normalization is used to the $i$ th objective vector $\boldsymbol{O}_{i}$ as

$\boldsymbol{O}_{i}^{\#}=\frac{\boldsymbol{O}_{i}-\min \left(\boldsymbol{O}_{i}\right)}{\max \left(\boldsymbol{O}_{i}\right)-\min \left(\boldsymbol{O}_{i}\right)}$,

where $\boldsymbol{O}_{i}^{\#}$ is the $i$ th objective vector after being normalized.

\subsubsection{Manual weight assignment strategy}

A manual weight $w_{\mathrm{m}}$ is introduced to establish a weighted objective according to operators' needs. Finally, the final objective $o$ is expressed as

$o=\sum_{i=1}^{k} w_{\mathrm{a}, i} w_{\mathrm{m}, i} O_{i}^{\#}$,

where $O_{i}^{\#}$ represents the value of the $i$ th objective, and $k$ represents the number of objectives.

\subsection{Optimization module}

The function of this module is to recommend wheel profiles that meet train operators' needs. This module consists of two parts: a response surface technique and an automatic optimization algorithm.

\subsubsection{RBF-based response surface technique}

For discrete datasets, parameter optimization is based on a large number of samples. Specifically, for optimization problems that rely on numerical simulations, a lot of repeated simulation runs are required [37, 38]. In the presented work, a single simulation takes nearly $3 \mathrm{~h}$ (Computing facilities: software: SIMPACK 2020X and MATLAB R2019a; hardware: Intel Core i7-4790 K, $4.00 \mathrm{GHz}$.), which is on the premise that the simulation can run smoothly. The calculation amount is so large that it is unrealistic to perform a large number of simulation runs, and an efficient and reliable method is of interest [39, 40]. In this paper, based on a small number of simulated samples, the RBF network is used as a regression tool to establish the relationship between the design variables $\left(\alpha_{1}, \alpha_{2}\right)$ described in Sect. 3.1 and the weighted objective (o) described in Sect. 3.3.

The RBF network [41, 42] is a feedforward neural network with a single hidden layer. RBF is the activation function of hidden neurons, and the output layer is a linear combination of the outputs of the hidden neurons. Given an input $\boldsymbol{\alpha}=\left[\alpha_{1}, \alpha_{2}\right]$, the RBF network produces a weighted sum output:

$o(\boldsymbol{\alpha})=\sum_{j=1}^{k} w_{j} \varphi_{j}\left(\boldsymbol{\alpha}, \boldsymbol{c}_{j}\right)+b$,

where $w_{j}$ is the weight, $b$ is the bias, $\boldsymbol{c}_{j}$ is the center of the $j$ th hidden neuron, and $k$ is the number of centers; $\varphi_{j}(\cdot)$ is the Gaussian RBF, expressed as

$\varphi_{j}\left(\boldsymbol{\alpha}, \boldsymbol{c}_{j}\right)=\exp \left(\frac{-\left\|\boldsymbol{\alpha}-\boldsymbol{c}_{j}^{2}\right\|}{2 \sigma_{j}^{2}}\right)$,

where $\sigma_{j}$ is the width of the $j$ th hidden neuron, and $\|*\|$ is the Euclidean norm.

\subsubsection{PSO for wheel profile recommendation}

It should be noted that this step is not necessary, because after obtaining the RBF-based response colormap, train operators can manually select the design point $\alpha=\left[\alpha_{1}, \alpha_{2}\right]$ according to the generated colormap to obtain one or more optimized wheel profiles. However, in this paper, PSO [43] is introduced to automatically find the optimal combination of $\alpha_{1}$ and $\alpha_{2}$, in which Eq. (19) is treated as the objective function of PSO. For a detailed description of the PSO method, as well as its application to railway wheel profile optimization, see Refs. [10, 43, 44].

\section{Application of WPFTS to wheel profile recommendation}

According to Eq. (18), we can know that the corresponding wheel profile can be generated only by entering the manual weight vector $\left(\boldsymbol{W}_{\mathrm{m}}\right)$ that meet the train operators' needs. Equation (18) can be rewritten as 
$o=\boldsymbol{W}_{\mathrm{a}} \cdot \boldsymbol{W}_{\mathrm{m}} \boldsymbol{O}_{\text {new }}^{\mathrm{T}}$,

where

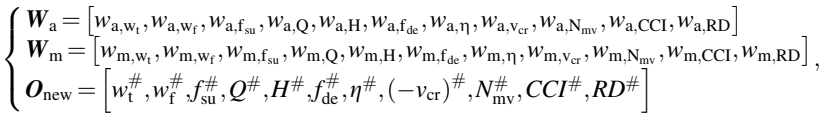

where $(\cdot)^{\#}$ represents the value of the objective after being normalized, and $(\cdot)^{\mathrm{T}}$ represents matrix transpose.

In WPFTS, based on the Latin hypercube sampling (LHS) strategy [45], a total of 79 sets of $\left(\alpha_{1}, \alpha_{2}\right)$ are selected in Module I to generate wheel profiles and are input into Module II to construct a sample dataset containing the 11 objectives listed in Fig. 1. Figure 9 shows the RBF-based responses $\left(w_{\mathrm{a}, i}, \boldsymbol{O}_{i}^{\#}\right)$ of some objectives. Figure 10a shows the RBF-based response that compromises all 11 objectives, i.e.,

$\boldsymbol{W}_{\mathrm{m}}=[1,1,1,1,1,1,1,1,1,1,1]$.

It should be noted that all wheel profiles derived from the dark blue region in the colormap can be considered as candidates. Figure $10 \mathrm{~b}$ shows the wheel profile corresponding to the design point $(1.010,1.020)$.

(a)

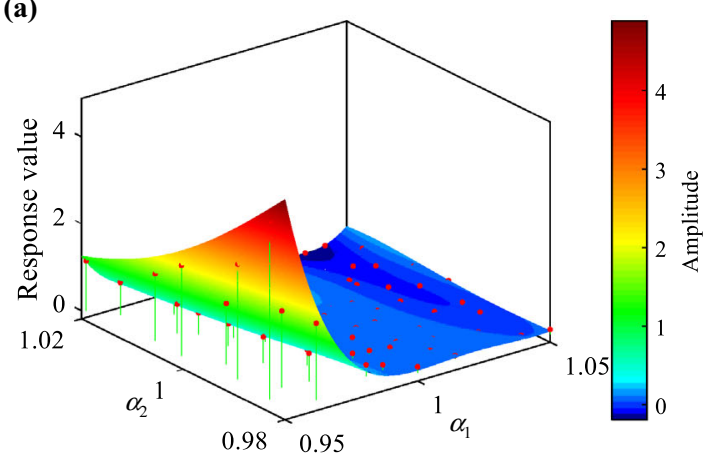

(c)

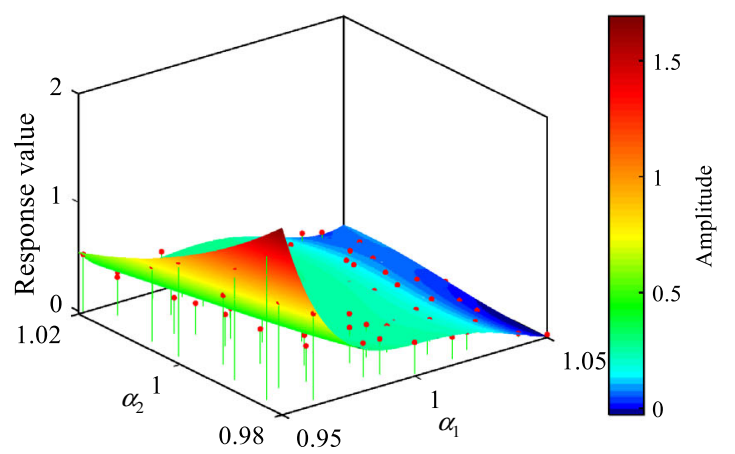

\subsection{Case I: application of WPFTS to produce a wheel profile with high shape stability}

Most of the existing references on wheel profile optimization use only short-term (or short-distance) MBS results to evaluate the dynamic performance of an optimized profile. However, the fact that the initial profile has better dynamic performance does not mean that the profile still has better dynamic performance as the mileage increases. The fundamental reason is that the shape of the wheel profile may be drastically destroyed. For example, in recent years, one topic that the German Centre for Rail Traffic Research has focused on is that: the 60E2 rail profile and regular maintenance of the rail profile, in conjunction with the usual wheel profiles, should result in a lower equivalent conicity compared with the 60E1 (UIC 60) rail profile [16], as shown in Fig. 11b. This brings good train running stability when the wheel profile is new. However, the contact point between the wheel and the rail, among other things, moves in a very small region, which favors the formation of hollow wear of railway wheels. As shown in Fig. 11c, d, when the interval of wheel lateral displacement is $[-5 \mathrm{~mm}, 5 \mathrm{~mm}$ ], the contact point movement range between the $\mathrm{S} 1002$ wheel and the 60E2 rail is much smaller than that between the $\mathrm{S} 1002$ wheel and the

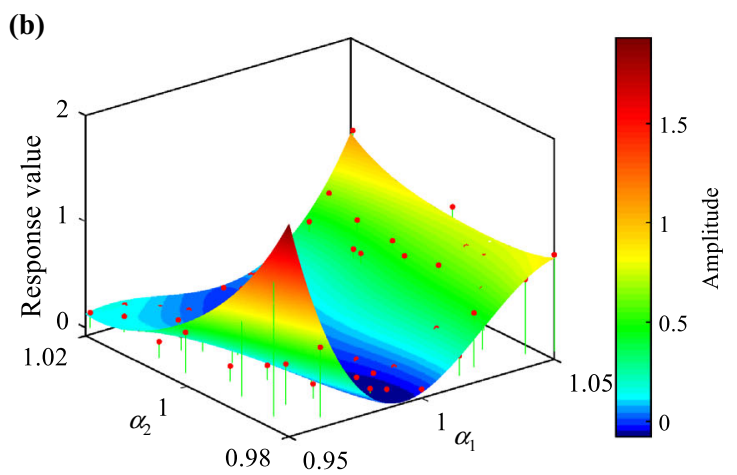

(d)

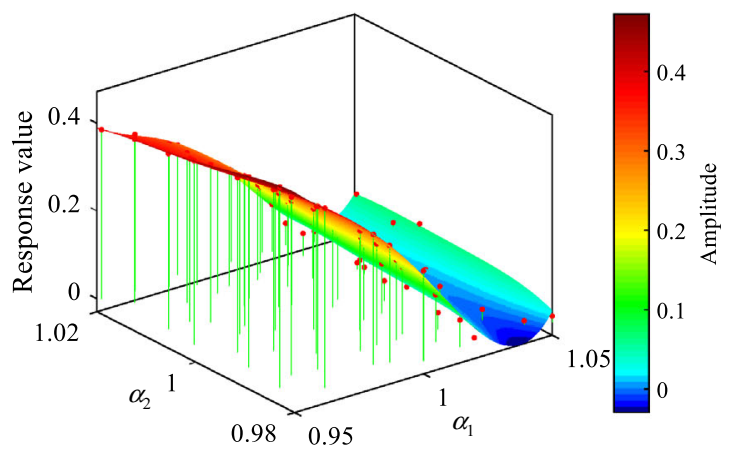

Fig. 9 RBF-based responses $\left(w_{\mathrm{a}, i}, \boldsymbol{O}_{i}^{\#}\right)$ of some objectives: a tread wear, b derailment coefficient, $\mathbf{c}$ ride comfort, and $\mathbf{d}$ critical speed 
(a)

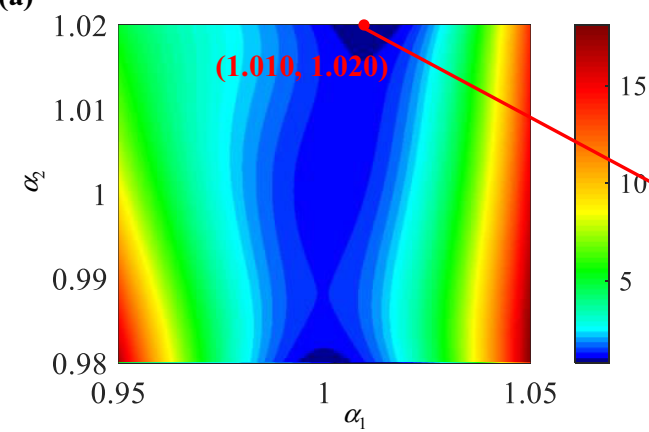

(b)

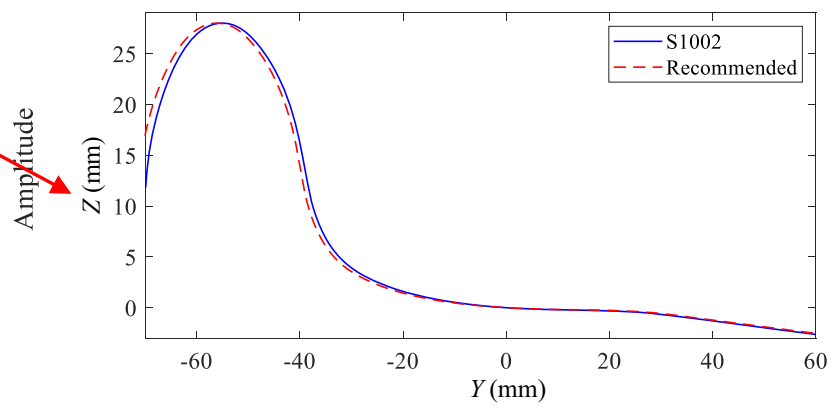

Fig. 10 The RBF-based response (a), and the optimal wheel profile recommended by WPFTS considering all 11 objectives (b)

$60 \mathrm{E} 1$ rail, where the rail cant is $1 / 40$, the wheelset back-toback distance is $1360 \mathrm{~mm}$, and the gauge measurement position is $14 \mathrm{~mm}$ below the rail crown. Consequently, a high equivalent conicity may occur and seriously deteriorate the train's stability. This phenomenon often occurs in high-speed trains. Therefore, a wheel profile with high shape stability is worthy of being developed.

On one hand, a wheel profile with high contact conformity is desirable because it makes the wear distribution on the tread region relatively uniform, thereby maintaining the shape stability. On the other hand, a smaller tread wear index and a smaller flange wear index contribute to good shape stability of the wheel profile. In the setting of $\boldsymbol{W}_{\mathrm{m}}$, the following principle is followed: a total weight value of 100 is assigned to the weights corresponding to the objectives being considered, and the weight value of an

(a)

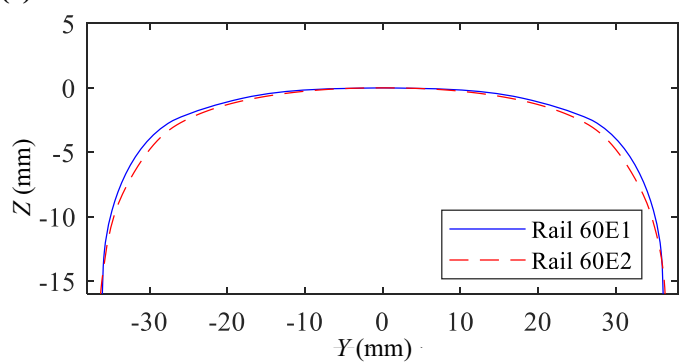

(c)

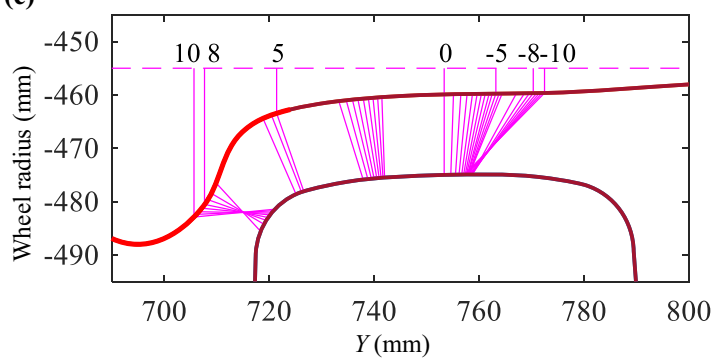

objective represents the importance of this objective. Therefore, a manual weight vector inputted into WPFTS for producing a wheel profile with high shape stability is set as

$\boldsymbol{W}_{\mathrm{m}}=[25,25,0,0,0,0,0,0,0,50,0]$.

Figure 12a shows the RBF-based response mainly considering CCI, $w_{\mathrm{t}}$, and $w_{\mathrm{f}}$; the profile S1002-SS shown in Fig. 12c is the wheel profile recommended by WPFTS. For comparison, the wheel profile S1002 and the wheel profile (S1002-W in Fig. 12b) corresponding to the minimum total wear index $\left(w=w_{\mathrm{f}}+w_{\mathrm{t}}\right.$ in Eq. (7)) in the short-term MBS (a trip on the virtual track listed in Table 1, i.e., a $15-\mathrm{km}$ distance) are introduced, where Eq. (22) is updated as

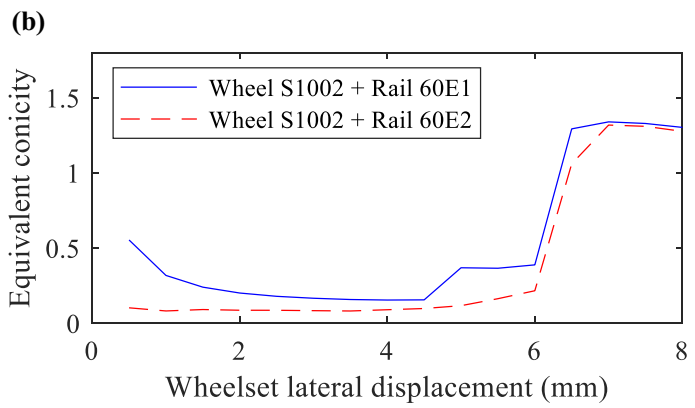

(d)

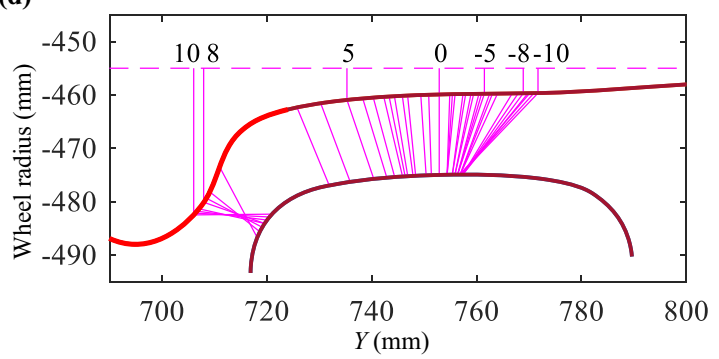

Fig. 11 Rail 60E1 and rail 60E2 (a), equivalent conicities for the 60E1 and 60E2 rail profiles that match the S1002 wheel profile (b), contact lines between the S1002 wheel and the 60E1 rail (c), and contact lines between the S1002 wheel and the 60E2 rail (d) 
$\left\{\begin{array}{l}\boldsymbol{W}_{\mathrm{a}, \mathrm{w}}=\left[w_{\mathrm{a}, \mathrm{w}}, w_{\mathrm{a}, \mathrm{f}_{\mathrm{su}}}, w_{\mathrm{a}, \mathrm{Q}}, w_{\mathrm{a}, \mathrm{H}}, w_{\mathrm{a}, \mathrm{f}_{\mathrm{de}},}, w_{\mathrm{a}, \eta}, w_{\mathrm{a}, v_{\mathrm{cr}}}, w_{\mathrm{a}, \mathrm{N}_{\mathrm{mv}}}, w_{\mathrm{a}, \mathrm{CCI}}, w_{\mathrm{a}, \mathrm{RD}}\right] \\ \boldsymbol{W}_{\mathrm{m}, \mathrm{w}}=\left[w_{\mathrm{m}, \mathrm{w}}, w_{\mathrm{m}, \mathrm{f}_{\mathrm{su}},}, w_{\mathrm{m}, \mathrm{Q}}, w_{\mathrm{m}, \mathrm{H}}, w_{\mathrm{m}, \mathrm{f}_{\mathrm{de}}}, w_{\mathrm{m}, \eta}, w_{\mathrm{m}, v_{\mathrm{cr}},}, w_{\mathrm{m}, \mathrm{N}_{\mathrm{mv}}}, w_{\mathrm{m}, \mathrm{CCI}}, w_{\mathrm{m}, \mathrm{RD}}\right], \\ \boldsymbol{O}_{\mathrm{new}, \mathrm{w}}=\left[w^{\#}, f_{\mathrm{su}}^{\#}, Q^{\#}, H^{\#}, f_{\mathrm{de}}^{\#}, \eta^{\#},\left(-v_{\mathrm{cr}}\right)^{\#}, N_{\mathrm{mv}}^{\#}, C C I^{\#}, R D^{\#}\right]\end{array}\right.$

and the manual weight vector $\boldsymbol{W}_{\mathrm{m}, \mathrm{w}}$ corresponding to the wheel profile $\mathrm{S} 1002-\mathrm{W}$ is expressed as

$\boldsymbol{W}_{\mathrm{m}, \mathrm{w}}=[100,0,0,0,0,0,0,0,0,0]$.

Figure 13 shows the wheel-rail contact lines and the equivalent conicities of the wheel profiles plotted in Fig. 12c that match the $60 \mathrm{E} 2$ rail profile; it indicates that the S1002-SS profile produces a more uniform wheel-rail contact distribution, while the S1002-W profile produces a more concentrated wheel-rail contact distribution. Figure 14 shows the wear distribution of the S1002 profile, the S1002-SS profile, and the S1002-W profile after the vehicle runs for $150000 \mathrm{~km}$ on the virtual track described in Table 1; it indicates that after a long-term operation, the S1002-SS profile produces the smallest wear depth and a more uniform wear distribution, while the S1002 profile and the S1002-W profile produce the largest wear depth in the tread region and in the flange region, respectively. Therefore, simultaneous consideration of the

(a)

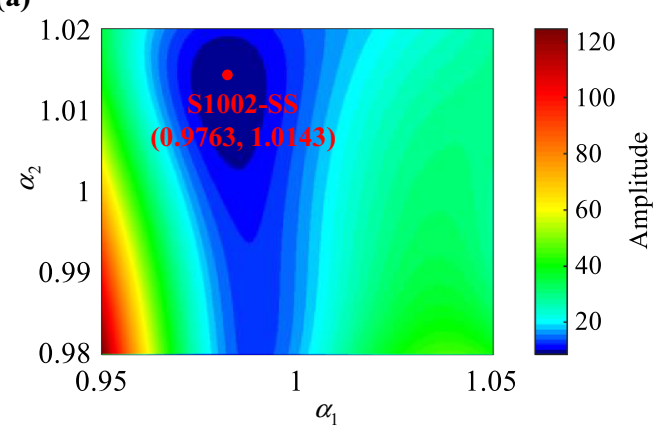

short-term tread wear index, short-term flange wear index, and CCI during the wheel profile design phase contributes to reducing wheel wear and improving the shape stability of the wheel profile during long-term operation. Besides, combined with the result shown in Fig. $12 \mathrm{~b}$, it can be concluded that the S1002-W wheel profile is optimized for better short-term total wear index but not for long-term wear performance, and only considering the total wear index may underestimate the damage to the wheel flange region.

\subsection{Case II: application of WPFTS to produce a wheel profile for mitigating the development of wheel OOR}

In this case, the purpose is to use WPFTS to generate a wheel profile that can mitigate the wheel OOR development, so we adjust the weights corresponding to and related to radial deviation.

Figure 15 shows the relationship between the tread wear index, the radial deviation, and the nominal equivalent conicity for the 79 sampled profiles that match the 60E2 rail profile. The wear index and the radial deviation shown in this figure are the cumulative tread wear number and the

(b)

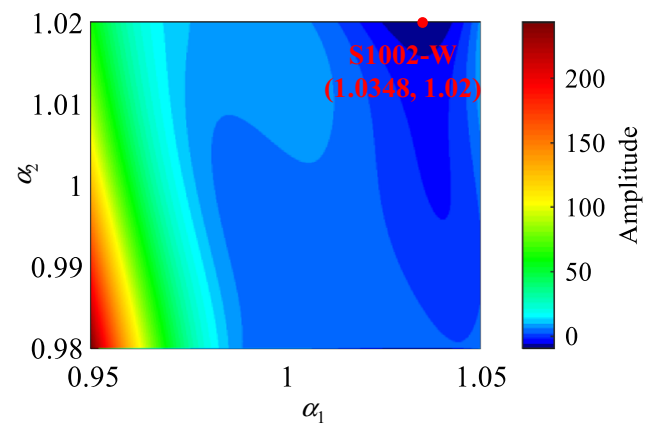

(c)

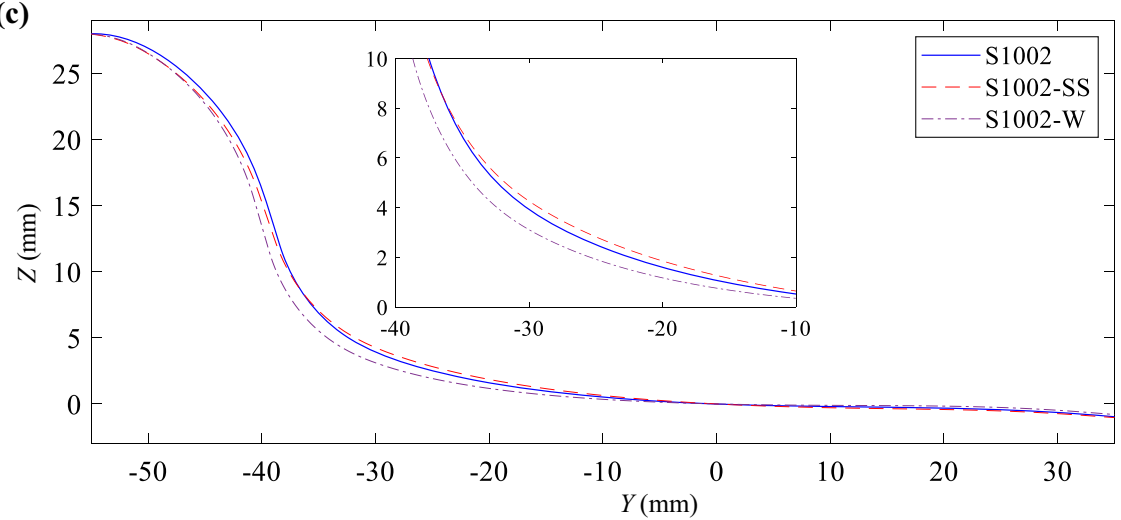

Fig. 12 The design point corresponding to the wheel profile with high shape stability (a), the design point corresponding to the wheel profile for reducing short-term wear index (b), and comparison between S1002, S1002-SS, and S1002-W profiles (c) 
(a)

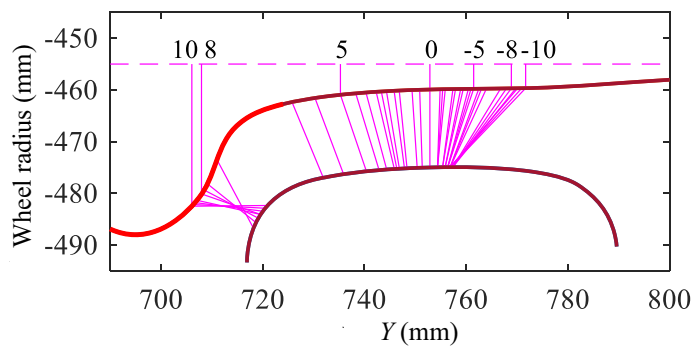

(c)

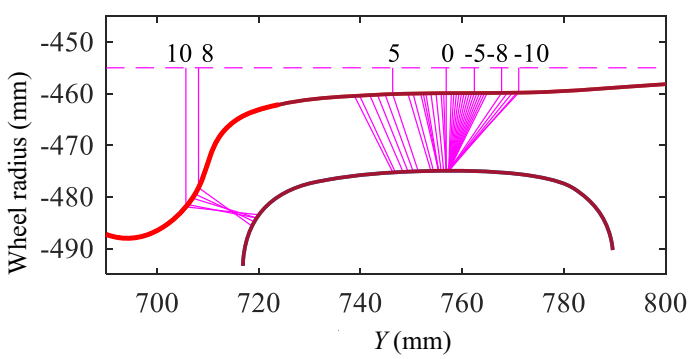

(b)

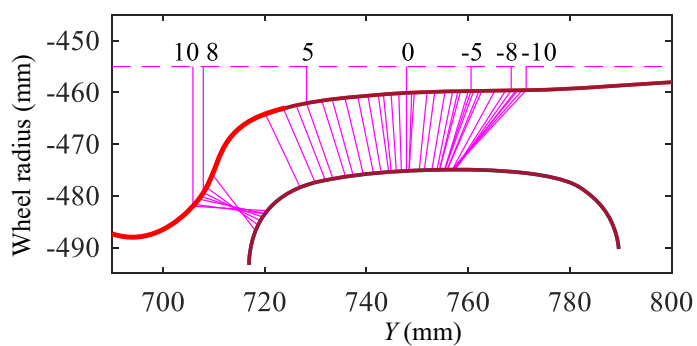

(d)

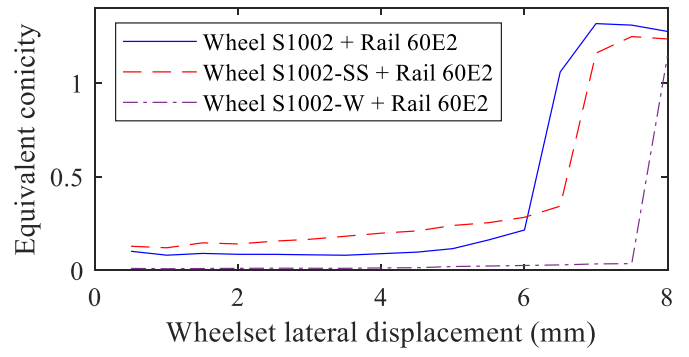

Fig. 13 Wheel-rail contact lines of S1002 + Rail 60E2 (a), S1002-SS + Rail 60E2 (b), S1002-W + Rail 60E2 (c), and comparison of equivalent conicities $(\mathbf{d})$

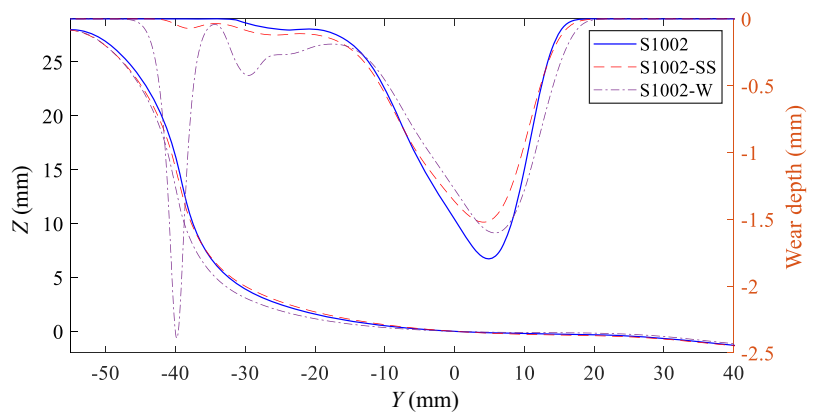

Fig. 14 Comparison of wear distributions (wheel wear calculation model: Hertzian-FASTSIM-Jendel [46]; updating strategy: wear depth-based, threshold $=0.1 \mathrm{~mm}$ [47]; smoothing strategy: moving average filter and LOWESS [48])

maximum radial deviation after 10 wheel revolutions described in Sect. 3.2.3, respectively. The nominal equivalent conicity is the equivalent conicity when the wheelset lateral displacement is $3 \mathrm{~mm}$. This figure illustrates that the equivalent conicity affects the development of wheel OOR. Roughly speaking, a wheel profile with a low nominal equivalent conicity brings a low tread wear index and low radial deviation. First, the following manual weight vector $\boldsymbol{W}_{\mathrm{m}}$ considering only RD is introduced:

$\boldsymbol{W}_{\mathrm{m}}=[0,0,0,0,0,0,0,0,0,0,100]$.

Figure 16a shows the RBF-based response considering only RD, and the recommended profile S1002-RD is shown in Fig. 16c.

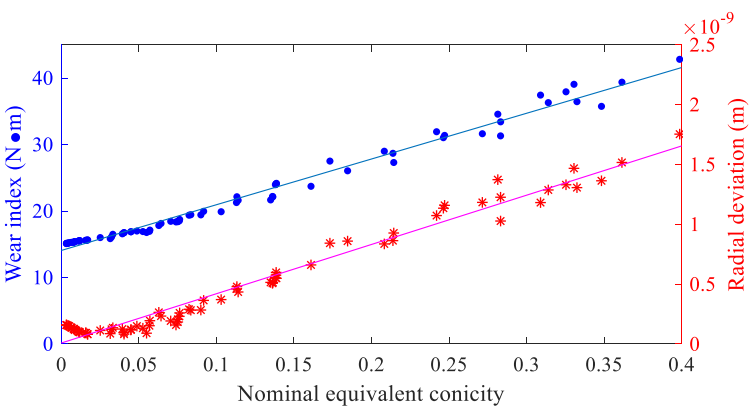

Fig. 15 The relationship between the wear index, the radial deviation, and the nominal equivalent conicity for the 79 sampled wheel profiles that match the $60 \mathrm{E} 2$ rail profile

As described in Sect. 4.1, shape stability should be also considered in designing a wheel profile. Furthermore, the manual weight vector that simultaneously takes into account RD and CCI is set as

$\boldsymbol{W}_{\mathrm{m}}=[0,0,0,0,0,0,0,0,0,50,50]$.

Figure $16 \mathrm{~b}$ shows the RBF-based response that takes into account $\mathrm{RD}$ and $\mathrm{CCI}$, and the recommended profile S1002-RDSS is shown in Fig. 16c. Figure 17 shows the equivalent conicities of S1002, S1002-RD, and S1002RDSS wheel profiles that match the $60 \mathrm{E} 2$ rail profile, where the rail cant is $1 / 40$, the wheelset back-to-back distance is $1360 \mathrm{~mm}$, and the gauge measurement position is $14 \mathrm{~mm}$ below the rail crown. We can see that the equivalent conicity of the S1002-RDSS profile lies between 
that of S1002 and S1002-RD wheel profiles. The simulation verification is omitted because it involves the establishment of a complex long-distance flexible track, which is unrealistic.

\subsection{Case III: application of WPFTS to produce a wheel profile considering hunting stability and derailment safety}

This section describes how to use WPFTS to recommend a wheel profile that simultaneously considers hunting stability (hunting speed $v_{\mathrm{cr}}$ ) and derailment safety $\left(f_{\mathrm{de}}\right)$. Correspondingly, the following manual weight vector is used:

$\boldsymbol{W}_{\mathrm{m}}=[0,0,0,0,0,50,0,50,0,0,0]$.

Figure 18a shows the RBF-based response considering $v_{\text {cr }}$ and $f_{\text {de }}$. The colormap shows that two optimal regions (blue areas) are generated, so two corresponding wheel profiles (S1002-P1 and S1002-P2) are recommended, as shown in Fig. 18c.

Considering that a wheel profile with good shape stability can maintain the initial dynamic performances of the train for longer, we further take CCI into consideration and the manual weight vector is set as

(a)

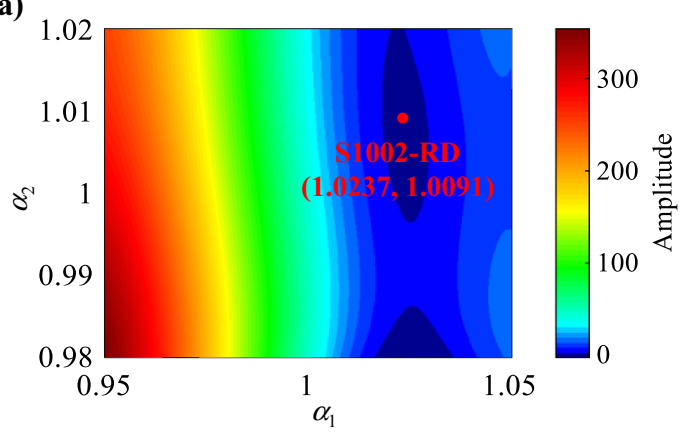

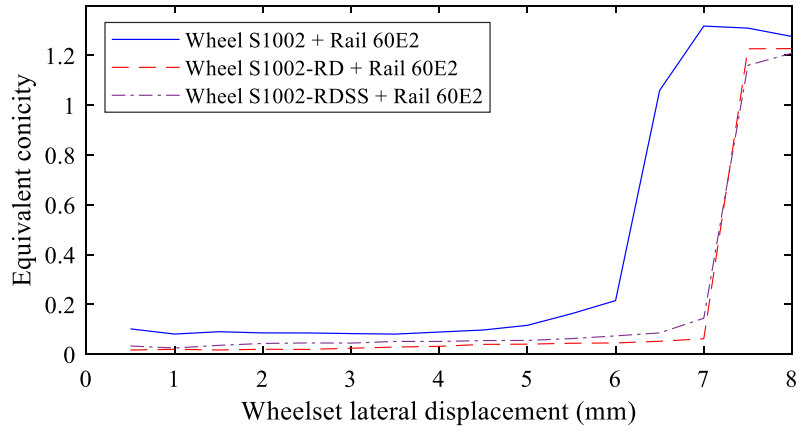

Fig. 17 Equivalent conicities of S1002, S1002-RD, and S1002-RDSS wheel profiles that match the $60 \mathrm{E} 2$ rail profile

$\boldsymbol{W}_{\mathrm{m}}=[0,0,0,0,0,33,0,33,0,33,0]$.

Figure $18 \mathrm{~b}$ shows the RBF-based response mainly considering $v_{\mathrm{cr}}, f_{\mathrm{de}}$, and $\mathrm{CCI}$, and the profile S1002-P3 shown in Fig. 18c is the wheel profile recommended by WPFTS. Finally, the equivalent conicity, critical speed, derailment coefficient, and contact concentration index corresponding to these four profiles are shown in Fig. 19 and Table 2, where the rail cant is $1 / 40$, the wheelset backto-back distance is $1360 \mathrm{~mm}$, and the gauge measurement position is $14 \mathrm{~mm}$ below the rail crown.

(b)

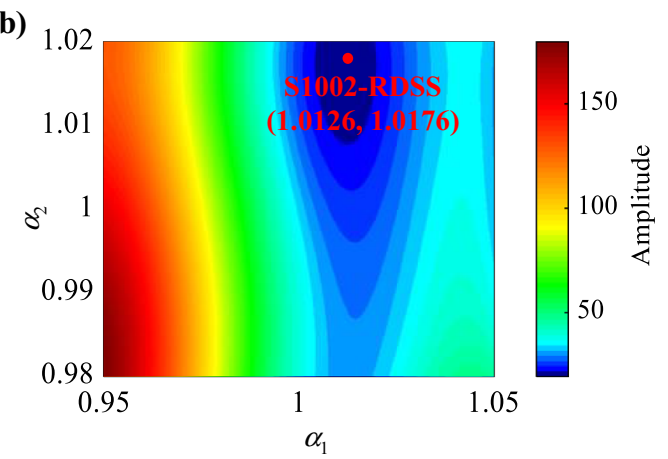

(c)

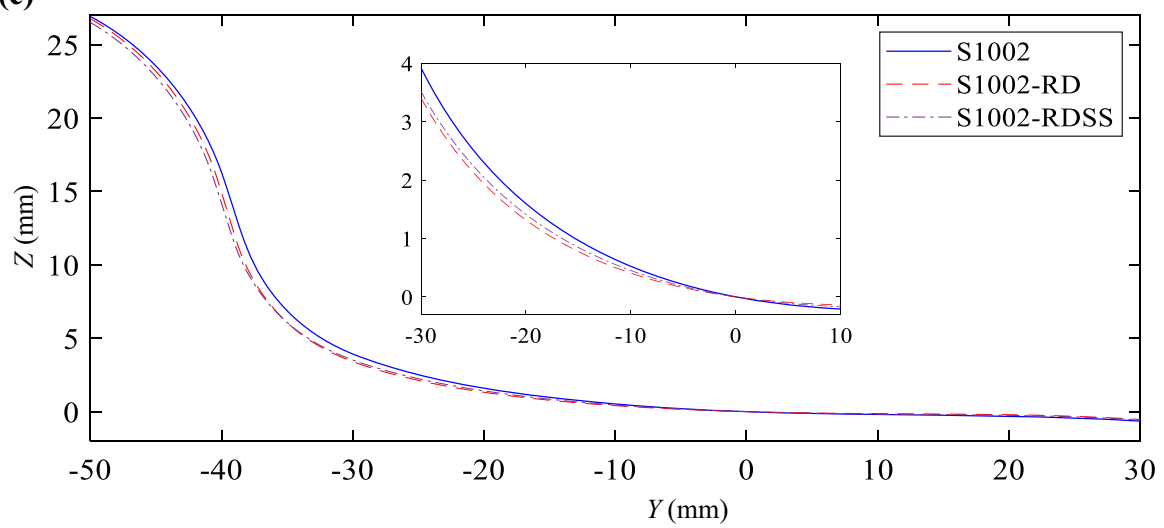

Fig. 16 RBF-based response considering RD (a), RBF-based response considering RD and CCI (b), and comparison between S1002, S1002-RD, and S1002-RDSS wheel profiles (c) 
(a)

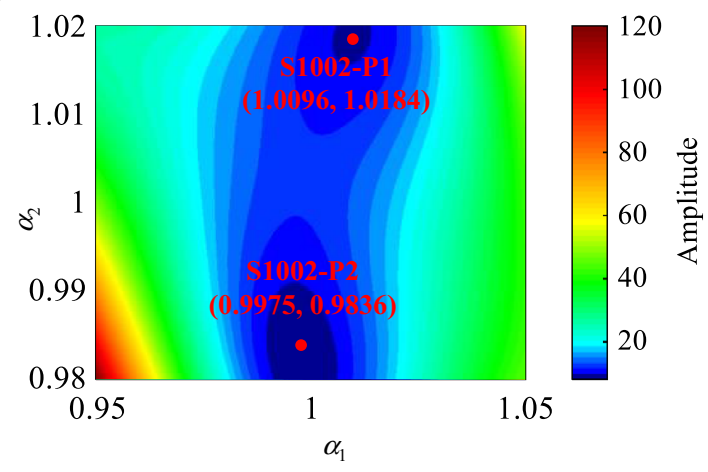

(b)

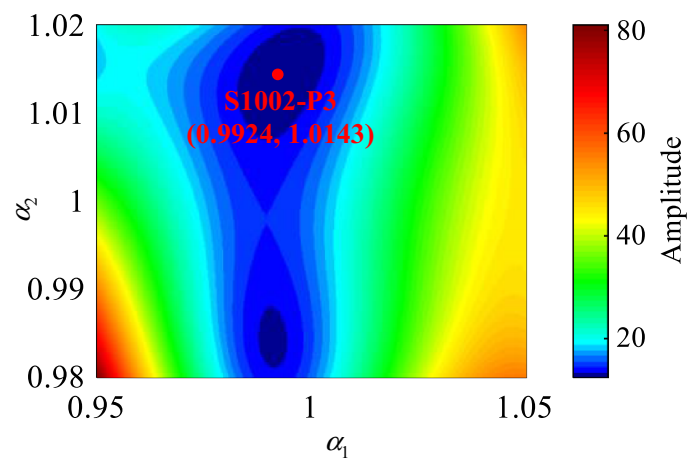

(c)

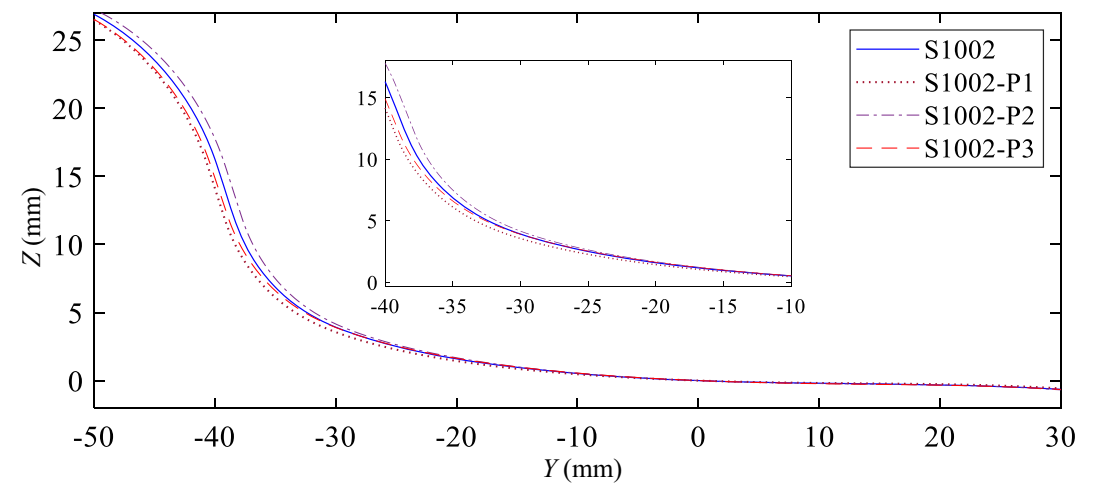

Fig. 18 RBF-based response considering $v_{\mathrm{cr}}$ and $f_{\mathrm{de}}(\mathbf{a})$, RBF-based response considering $v_{\mathrm{cr}}, f_{\mathrm{de}}$, and CCI (b), and the optimal wheel profile recommended by WPFTS (c)

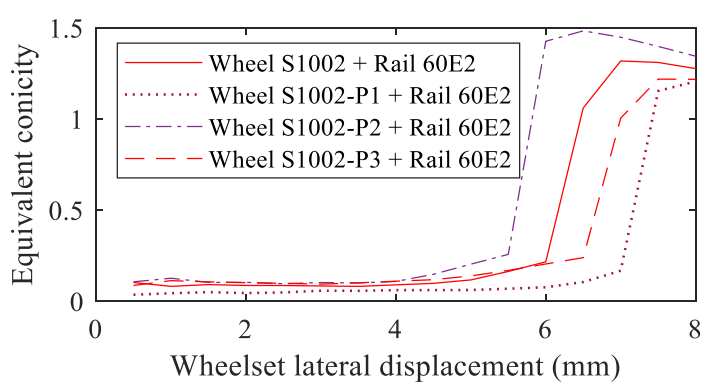

Fig. 19 Equivalent conicities of S1002, S1002-P1, S1002-P2, and S1002-P3 wheel profiles that match the 60E2 rail profile

\section{Conclusions and discussion}

In this paper, we develop a WPFTS that comprehensively considers the influence of wheel profiles on maintenancerelated wheel wear (transversal and circumferential) and wheel surface fatigue, vehicle stability, vehicle safety, and passenger comfort. WPFTS can recommend one or more optimized wheel profiles according to train operators' needs, e.g., mitigating the development of wheel OOR, improving ride comfort, reducing wheel wear, improving the shape stability of the wheel profile. Specifically, WPFTS consists of the following four modules:
I. Wheel profile generation module This module is used for producing candidate profiles using the RSFT method proposed in Ref. [1].

II. Multi-objective generation module This module is used to generate the objective database and consists of three models: (a) A rigid MBS model for generating tread wear index $w_{\mathrm{t}}$, flange wear index $w_{\mathrm{f}}$, wheel surface fatigue $f_{\mathrm{su}}$, wheel-rail vertical force $Q$, sum of wheelset lateral forces $H$, derailment coefficient $f_{\text {de }}$, overturning coefficient $\eta$, mean comfort index $N_{\mathrm{mv}}$, and train critical speed $v_{\mathrm{cr}}$. (b) An analytical model for producing CCI proposed by Polach [13]. It should be noted that the CCI is enhanced based on the MBS results. (c) A rigid-flexible coupled MBS model for generating wheel radial deviation RD.

III. Weight assignment module An adaptive weight assignment strategy that takes into account the sensitivity of an optimization objective to changes in wheel profile and a manual weight assignment strategy based on the train operators' specific requirements are introduced.

IV. Optimization module A response surface technique (i.e., RBF) and a bio-inspired optimization algorithm (i.e., PSO) are combined to quickly and reliably 
Table 2 Critical speed $v_{\text {cr }}$, derailment coefficient $f_{\text {de }}$, and CCI for S1002, S1002-P1, S1002-P2, and S1002-P3 wheel profiles

\begin{tabular}{llll}
\hline Profile & Hunting speed $v_{\text {cr }}(\mathrm{km} / \mathrm{h})$ & Derailment coefficient $f_{\text {de }}$ & CCI \\
\hline S1002 & 275.40 & 0.1163 & 0.0371 \\
S1002-P1 & 292.61 & 0.1168 & 0.0378 \\
S1002-P2 & 272.13 & 0.1017 & 0.0375 \\
S1002-P3 & 263.07 & 0.1101 & 0.0315 \\
\hline
\end{tabular}

recommend an optimal wheel profile according to train operators' needs.

Based on the Manchester Benchmark passenger vehicle, three cases are introduced to show how WPTFS recommends a wheel profile according to train operators' needs. Among them, a wheel profile with high shape stability, a wheel profile for mitigating the development of wheel OOR, and a wheel profile considering hunting stability and derailment safety are developed, respectively. Based on simulation results, the following notes are listed here:

I With the help of WPFTS, by only entering the manual weight vector, train operators can automatically obtain one or more wheel profiles that meet their needs.

II The fact that the initial profile has better dynamic performance does not mean that the profile still has better dynamic performance as the mileage increases. Therefore, in wheel profile design, it is recommended to consider a wheel profile with high shape stability, where CCI can be regarded as an evaluation index.

This paper ends with the following notes:

I The WPFTS is developed based on the Manchester Benchmark passenger vehicle running on a virtual track. In order to get more realistic results, a more detailed vehicle model, as well as a representative route being closer to real-life operations, are required to update the objective database.

II It should be noted that the superiority of the optimized wheel profile relies heavily on the baseline wheel profile because the RSFT method only fine-tunes the wheel profile. In order to maximize the advantages of the optimized wheel profile, more baseline wheel profiles (e.g., the EPS and the 1/40 profiles specified in standard [17], the LM series and JM series profiles specified in standard [49]) can be input into the RSFT method to generate more candidate profiles.

III When optimizing specific objectives, other parameters related to safety and stability (referred to as objectives in this paper) should be set as constraints that satisfy the requirements specified in the standard [21]. In the case analyzed in this paper, all wheel profiles generated by the RSFD method $\left(0.95 \leq \alpha_{1} \leq 1.05\right.$ and $0.98 \leq \alpha_{2} \leq 1.02$ ) satisfy the specified criteria, so the setting of constraints is not mentioned.

IV In the face of strong simplifications of the simulation model, whether the final optimized wheel profile in real life still has an advantage needs to be further verified. Therefore, simulation acceptance according to standards and field experimental verification, such as mentioned in Ref. [50], are further required.

$\mathrm{V}$ The selection of the weight vector is the key to determine the recommended wheel profile. More systematic simulations and field experiments are needed to determine a reasonable weight vector.

Acknowledgments This work was supported by China Scholarship Council (Grant No. 201707000113). The authors would like to thank Prof. Oldrich Polach for his guidance on the contact concentration index (CCI) used in this paper. The authors would like to thank the anonymous reviewers for their comments that contributed to the work being substantially improved.

Open Access This article is licensed under a Creative Commons Attribution 4.0 International License, which permits use, sharing, adaptation, distribution and reproduction in any medium or format, as long as you give appropriate credit to the original author(s) and the source, provide a link to the Creative Commons licence, and indicate if changes were made. The images or other third party material in this article are included in the article's Creative Commons licence, unless indicated otherwise in a credit line to the material. If material is not included in the article's Creative Commons licence and your intended use is not permitted by statutory regulation or exceeds the permitted use, you will need to obtain permission directly from the copyright holder. To view a copy of this licence, visit http://creativecommons. org/licenses/by/4.0/.

\section{References}

1. Ye Y, Qi Y, Shi D, Sun Y, Zhou Y, Hecht M (2020) Rotaryscaling fine-tuning (RSFT) method for optimizing railway wheel profiles and its application to a locomotive. Railw Eng Sci 28(2):160-183

2. Shen G, Ayasse JB, Chollet H, Pratt I (2003) A unique design method for wheel profiles by considering the contact angle function. Proc Inst Mech Eng Part F J Rail Rapid Transit 217(1):25-30 
3. Shevtsov I, Markine V, Esveld C (2005) Optimal design of wheel profile for railway vehicles. Wear 258(7-8):1022-1030

4. Jahed H, Farshi B, Eshraghi MA, Nasr A (2008) A numerical optimization technique for design of wheel profiles. Wear 264(1-2):1-10

5. Polach O (2011) Wheel profile design for target conicity and wide tread wear spreading. Wear 271(1-2):195-202

6. Cui D, Li L, Jin X, Li X (2011) Optimal design of wheel profiles based on weighed wheel/rail gap. Wear 271(1-2):218-226

7. Shevtsov I, Markine V, Esveld C (2008) Design of railway wheel profile taking into account rolling contact fatigue and wear. Wear 265(9-10):1273-1282

8. Spangenberg U, Fröhling RD, Els PS (2018) Long-term wear and rolling contact fatigue behaviour of a conformal wheel profile designed for large radius curves. Veh Syst Dyn 57(1):44-63

9. Persson I, Iwnicki SD (2004) Optimisation of railway profiles using a genetic algorithm. Veh Syst Dyn 41:517-527

10. Lin F, Zhou S, Dong X, Xiao Q, Zhang H, Hu W et al (2021) Design method of LM thin flange wheel profile based on NURBS. Veh Syst Dyn 59(1):1-16

11. Firlik B, Staśkiewicz T, Jaśkowski W, Wittenbeck L (2019) Optimisation of a tram wheel profile using a biologically inspired algorithm. Wear 430-431:12-24

12. Ye Y, Sun Y, Dongfang S, Shi D, Hecht M (2020) Optimizing wheel profiles and suspensions for railway vehicles operating on specific lines to reduce wheel wear: a case study. Multibody Syst Dyn 51:91-122

13. Polach O, Nicklisch D (2016) Wheel/rail contact geometry parameters in regard to vehicle behaviour and their alteration with wear. Wear 366-367:200-208

14. BS EN 15313 (2016) Railway applications. In-service wheelset operation requirements. In-service and off-vehicle wheelset maintenance

15. Iwnick S (1998) Manchester benchmarks for rail vehicle simulation. Taylor \& Francis Group, London

16. DIN EN 13674-1 (2017) Railway applications-Track-RailPart 1: Vignole railway rails $46 \mathrm{~kg} / \mathrm{m}$ and above (includes Amendment A1:2017)

17. EN 13715:2006 (2006) Railway applications-wheelsets and bogies-wheels — Wheels tread, CEN Brussels, February 2006

18. Lewis R, Dwyer-Joyce RS (2004) Wear mechanisms and transitions in railway wheel steels. Proc Inst Mech Eng Part J J Eng Tribol 218(6):467-478

19. Kabo E, Ekberg A, Torstensson PT, Vernersson T (2010) Rolling contact fatigue prediction for rails and comparisons with test rig results. Proc Inst Mech Eng Part F J Rail Rapid Transit 224(4):303-317

20. Choi H-Y, Lee D-H, Lee J (2013) Optimization of a railway wheel profile to minimize flange wear and surface fatigue. Wear 300(1-2):225-233

21. UIC Code 518 OR (2009) Testing and approval of railway vehicle from the point of view of their dynamic behavioursafety - track fatigue - running behavior, International Union of Railway

22. EN 12299 (2009) Railway applications-ride comfort for passengers-measurements and evaluation. Brussels: CEN

23. Polach O (2006) On non-linear methods of bogie stability assessment using computer simulations. Proc Inst Mech Eng Part F J Rail Rapid Transit 220(1):13-27

24. Vuitton J (2018) Nichtlineare Fahrwerkdynamik an der Stabilitätsgrenze. Dissertation, Technische Universität, Berlin

25. King F (2009) The Hilbert transform of waveforms and signal processing. In: Hilbert Transforms (Encyclopedia of Mathematics and its Applications, pp 119-181. Cambridge: Cambridge University Press. https://doi.org/10.1017/CBO9780511735271. 005 .
26. Polach O (2020) Contact distribution: criteria for assessing and optimizing wheel/rail profile pairing. In: International rail vehicle conference, Dresden, pp 45-47. http://polach.ch/publikationen. php?lang=de

27. Wang K (1984) The track of wheel contact points and the calculation of wheel/rail geometric contact parameters. J Southwest Jiaotong Univ 19(1):88-99 (in Chinese)

28. Tao G, Wen Z, Jin X, Yang X (2020) Polygonisation of railway wheels: a critical review. Railw Eng Sci 28(4):317-345

29. Zhai W, Jin X, Wen Z, Zhao X (2020) Wear problems of highspeed wheel/rail systems: observations, causes, and countermeasures in China. Appl Mech Rev 72(6):060801

30. Kalousek J, Johnson KL (1992) An investigation of short pitch wheel and rail corrugations on the Vancouver mass transit system. Proc Inst Mech Eng Part F J Rail Rapid Transit 206(2):127-135

31. Morys B (1999) Enlargement of out-of-round wheel profiles on high speed trains. J Sound Vib 227(5):965-978

32. Tao G, Xie C, Wang H, Yang X, Ding C, Wen Z (2020) An investigation into the mechanism of high-order polygonal wear of metro train wheels and its mitigation measures. Veh Syst Dyn. https://doi.org/10.1080/00423114.2020.1770810

33. Peng B, Iwnicki S, Shackleton P, Zhao Y, Cui D (2005) A practical method for simulating the evolution of railway wheel polygonalization. In: Proceedings of the 25th international symposium on dynamics of vehicles on roads and tracks (IAVSD 2017), 2017 August 14-18; Rockhampton, Queensland, Australia, pp 753-758

34. Qu S, Zhu B, Zeng J, Dai H, Wu P (2020) Experimental investigation for wheel polygonisation of high-speed trains. Veh Syst Dyn. https://doi.org/10.1080/00423114.2020.1772984

35. Ma C, Gao L, Cui R, Xin T (2021) The initiation mechanism and distribution rule of wheel high-order polygonal wear on highspeed railway. Eng Fail Anal 119:104937

36. Ye Y, Shi D, Krause P, Tian Q, Hecht M (2019) Wheel flat can cause or exacerbate wheel polygonization. Veh Syst Dyn 58(10): 1575-1604

37. Ye Y, Shi D, Poveda-Reyes S, Hecht M (2020) Quantification of the influence of rolling stock failures on track deterioration. J Zhejiang Univ Sci A 21(10):783-798

38. Ye Y, Sun Y (2020) Reducing wheel wear from the perspective of rail track layout optimization. Proc Inst Mech Eng Part K J Multi-body Dyn. https://doi.org/10.1177/1464419320956831

39. Ye Y, Shi D, Krause P, Hecht M (2019) A data-driven method for estimating wheel flat length. Veh Syst Dyn 58(9):1-19

40. Pandey M, Regis RG, Datta R, Bhattacharya B (2020) Surrogateassisted multi-objective optimization of the dynamic response of a freight wagon fitted with three-piece bogies. Int J Rail Transp. https://doi.org/10.1080/23248378.2020.1792808

41. Schwenker F, Kestler HA, Palm G (2001) Three learning phases for radial-basis-function networks. Neural Netw 14(4-5):439-458

42. Faris H, Aljarah I, Mirjalili S (2017) Evolving radial basis function networks using Moth-Flame Optimizer. Handb Neural Comput. https://doi.org/10.1016/b978-0-12-811318-9.00028-4

43. Kennedy J, Eberhart R (1995) Particle swarm optimization. In: Proceedings of IEEE international conference on neural networks 4:1942-1948. doi:https://doi.org/10.1109/ICNN.1995.488968

44. Cui D, Wang R, Allen P, An B, Li L, Wen Z (2018) Multiobjective optimization of electric multiple unit wheel profile from wheel flange wear viewpoint. Struct Multidiscip Optim 59(1):279-289

45. Roshanian J, Ebrahimi M (2013) Latin hypercube sampling applied to reliability-based multidisciplinary design optimization of a launch vehicle. Aerosp Sci Technol 28(1):297-304 
46. Jendel T (2002) Prediction of wheel profile wear-comparisons with field measurements. Wear 253(1-2):89-99

47. Barbarino G (2004) A fast and reliable mathematical model for the prediction of railway wheel wear. Dissertation, Politecnico di Milano

48. Ye Y, Sun Y, Shi D, Peng B, Hecht M (2021) A wheel wear prediction model of non-Hertzian wheel-rail contact considering wheelset yaw: comparison between simulated and field test results, Wear. https://doi.org/10.1016/j.wear.2021.203715

49. TB/T 449-2016 (2016) Wheel profile for locomotive and car (Chinese Standard in English), Issued by National Railway Administration

50. Götz G (2018) Model validation in rail vehicle technology for driving approval. Berlin: Technical University of Berlin 